\title{
Complexes of tetraethylthiuram disulphide with group 12 metals: single-source precursor in metal sulphide nanoparticles' synthesis
}

\author{
Satyendra N. Shukla $\cdot$ Pratiksha Gaur • \\ Nidhi Rai
}

Received: 26 July 2014/ Accepted: 19 August 2014/Published online: 7 September 2014

(C) The Author(s) 2014. This article is published with open access at Springerlink.com

\begin{abstract}
Six new complexes of $\mathrm{Zn}$ (II), $\mathrm{Cd}$ (II) and $\mathrm{Hg}$ (II) with sulphur containing ligand, tetraethylthiuram disulphide in 2:2 and 1:4 ratio have been synthesized. The resulting complexes have been characterized on the basis of molar conductance measurement, elemental analyses, FT-IR, ${ }^{1} \mathrm{H}-\mathrm{NMR}$, and FAB/ESI-mass studies. The complexes were used as a single-source precursor for the synthesis of $\mathrm{ZnS} / \mathrm{CdS} / \mathrm{HgS}$ nanoparticles by their thermal decomposition in the presence of different surfactants. The precursor:surfactant ratio and temperature play an important role in determining the size of the nanoparticles. The size and morphology of nanoparticles have been ascertained by ultra violet-visible spectroscopy, X-ray diffraction measurements and transmission electron microscopy. Schiff bases, complexes and nanoparticles were tested for antibacterial activity and minimum inhibitory concentration values against Escherichia coli. The complexes were found more potent than the corresponding Schiff bases and nanoparticles.
\end{abstract}

Keywords Metal sulphide nanoparticles - Thermal decomposition · Disulfiram $\cdot \mathrm{X}$-ray diffraction .

Transmission electron microscopy

Electronic supplementary material The online version of this article (doi:10.1007/s13204-014-0351-0) contains supplementary material, which is available to authorized users.

S. N. Shukla $(\varangle) \cdot$ P. Gaur · N. Rai

Coordination Chemistry Research Lab, Department

of Chemistry, Government Model Science College,

Jabalpur 482 001, MP, India

e-mail: ccrl_2004@rediffmail.com; sns1963_1@rediffmail.com

\section{Introduction}

Recent progress in nanotechnology allows for the creation of new materials with properties tunable on the nanometer scale. Applications range from using fluorescent nanoparticles as dyes for fluorescence labelling of cells and for motility assays, using gold nanoparticles for immune staining and gene delivery and using magnetic nanoparticles as contrast agents for magnetic resonance imaging and for hypothermia (Wu et al. 2003; Dahan et al. 2003; Lidke et al. 2004). In addition to this, nanotechnology provides products with specified and unique properties, which are finding application in various branches of electronic, chemical, food and textile industry as well as in medicine, pharmacy, agriculture, architectural engineering, aviation and in defense. It provides tools which allow to identifying changes and taking repair operations on cellular and molecular level and applying therapy oriented for specific structures in cell.

Semiconductor nanoparticles show a characteristic physicochemical property that differs from both the bulk and the molecular substance. These properties open up their use in diverse fields, such as optoelectronics, solar energy conversion and photocatalysis. As a common semiconductor material, IIB transition metal chalcogenides have attracted much attention due to their excellent properties and wide range of potential applications (Hoffmann et al. 1995; Brus 1991). These nanoparticles are important semiconductor compounds and one of the most explored because of their wide range of potential applications. $\mathrm{ZnS}$ has a wide direct band gap of $3.65 \mathrm{eV}$ (bulk) and it is a promising material for optoelectronic device applications such as optical coatings, solid-state solar cell windows, electrooptic modulators, photoconductors, field-effect transistors, sensors, transductors, light-emitting applications, and photonic 
crystal devices which operate in the region from visible to near-infrared. CdS nanoparticles are the most studied system amongst all the II-VI semiconducting nanoparticles. Bulk CdS has a direct band gap of $2.42 \mathrm{eV}$ at $300 \mathrm{~K}$ and a typical Bohr exciton diameter of around $5.8 \mathrm{~nm}$. As a result, in the size range of 1-6 nm, CdS nanoparticles show sizable quantum confinement effects. Cadmium sulphide shows great potential for uses in photochemical catalysis, solar cells, and nonlinear optical materials, and could be used as bioorganic detector of proteins or DNA. Nanocrystalline $\mathrm{HgS}$ has pronounced dichroism, photoelectric, acoustooptic properties and electrostatic image properties. Thus, it is a well-known technologically important material. Despite these applications of $\mathrm{HgS}$ nanoparticles, relatively less study is available due to difficulty in synthesis and toxicity of mercury (Onwudiwe and Ajibadercury 2011).

In recent years, metal compounds with $\mathrm{d}^{10}$ electronic configuration have received a lot of attention in the fields of inorganic chemistry, biochemistry and environmental chemistry. About 20 zinc enzymes are known in which zinc is generally tetrahedrally four-coordinate and bonded to hard donor atoms such as nitrogen (Marchettic et al. 1999). Previously, it has been reported that $\mathrm{Zn}$ (II) and $\mathrm{Cd}$ (II) complexes with Schiff base-type chelating ligand can be used as an effective emitting layer and showed quite good photo-physical properties (Kawamoto et al. 2008). Zinc complexes have been shown to be active as antitumor, antiHIV and antimicrobial agents (Sheng et al. 2008; Kaczmareka et al. 2009; Cowley et al. 2005; Koike et al. 1996).

Disulfiram is a drug used to support the treatment of chronic alcoholism by producing an acute sensitivity to alcohol and treatment for cocaine dependence, as it prevents the breakdown of dopamine (a neurotransmitter whose release is stimulated by cocaine); the excess dopamine results in increased anxiety, higher blood pressure, restlessness and other unpleasant symptoms. Several studies have reported that it has anti-protozoal activity as well (Nash and Rice 1998; Bouma et al. 1998). Disulfiram was also chosen as a payload with micelle and liposome nanoparticles on a mouse fibroblast cell L929. The toxic effect observed was most likely not due to the disulfiram delivered by the nanoparticles but rather to the amount of free disulfiram that is present in the nanoparticle preparation (Lobler et al. 2009).

Therefore, in anticipation of an interesting chemistry and biological activity, we have synthesized tetraethylthiuram disulphide (TETD) complexes of $\mathrm{Cd}(\mathrm{II}), \mathrm{Zn}$ (II) and $\mathrm{Hg}(\mathrm{II})$ in different molar ratio and characterized them by spectroscopic methods. These complexes were further used as a single-source precursor for the thermal synthesis of metal sulphide nanoparticles. The actual aim of this work is to validate how TETD metal derivative could be used as single-source precursor for the thermal synthesis of metal sulphide nanoparticles and to study and compare the antibacterial activity of the Schiff bases, complexes and nanoparticles synthesized.

\section{Materials and methods}

All the chemicals and solvents were used of A. R. grade. Mercury (II) chloride, cadmium (II)chloride, tri-octylphosphine oxide, triethanolamine, oleic acid, methanol, toluene, TETD (disulfiram) (All E. Merck), hexadecylamine (HDA) (Aldrich), zinc(II)chloride (CDH) were used as received. Muffle furnace with digital temperature control was used for thermal decomposition of complexes. Elemental analysis $(\mathrm{C}, \mathrm{H}, \mathrm{N}, \mathrm{S})$ was performed on Elementra Vario EL III Elemental Analyzer. Molar conductance measurement of complexes was carried out in EI-181 Conductivity Bridge with dipping-type cell at $25{ }^{\circ} \mathrm{C}$. Shimadzu-1700, ultra violet-visible (UV-Vis) spectrophotometer was used to carry out the optical measurements and the samples were placed in silica cuvettes $(1 \mathrm{~cm}$, path length), using toluene as a reference solvent. Fourier transform infrared spectra were recorded in $\mathrm{KBr}$ pellets on Shimadzu-8400 PC, FT-IR spectrophotometer. ${ }^{1} \mathrm{H}$-nuclear magnetic resonance spectra were recorded in MeOD on a Bruker DRX-300 NMR spectrometer. Electrospray ionization mass spectra were recorded on Micromass Quattro II mass spectrometer. X-ray diffraction (XRD) measurements were carried out using $\mathrm{Cu}-\mathrm{K} \alpha$ radiation $(\lambda=0.154 \mathrm{~nm})$ with Bruker D8-Advance X-ray diffractometer and graphs were plotted using Origin software. Transmission electron microscopy photographs were taken on a Tecnai $20 \mathrm{G} 2$ and Image $\mathbf{J}$ software was used for the calculation of d-spacing. Pcpdfwin software was used for matching calculated $\mathrm{d}$-spacing with standard values of $\mathrm{d}$ and hkl reflection planes.

Synthesis of complexes

Synthesis of $\left[\mathrm{Zn}(\mathrm{TETD}) \mathrm{Cl}_{2}\right]_{2}$, complex 1

Zinc chloride $(0.273 \mathrm{~g}, 0.002 \mathrm{~mol})$ was dissolved in $40 \mathrm{~mL}$ of ethanol in a two-neck flask. The ligand TETD, $(0.593 \mathrm{~g}$, $0.002 \mathrm{~mol}$ ), dissolved in $100 \mathrm{~mL}$ of ethanol, was added to the above solution and stirred for $2-3 \mathrm{~h}$. Thereafter, zinc chloride ( $0.1366 \mathrm{~g}, 0.001 \mathrm{~mol})$ dissolved in $20 \mathrm{~mL}$ ethanol, was added to above reaction mixture. A light grey precipitate was obtained after refluxing $15-20 \mathrm{~h}$ in an inert atmosphere, which was filtered, vacuum dried at room temperature and recrystallized from ethanol:acetone:chloroform, 1:1:2 (v/v) solvent mixture. Yield $(0.796 \mathrm{~g}$, $91.94 \%$ ), mp $120-125{ }^{\circ} \mathrm{C}$. found: $\mathrm{C}, 27.72 ; \mathrm{H}, 4.66$; , 
6.41; S, $29.61 \% . \mathrm{C}_{20} \mathrm{H}_{40} \mathrm{Cl}_{4} \mathrm{~N}_{4} \mathrm{~S}_{8} \mathrm{Zn}_{2}$ requires: $\mathrm{C}, 27.75 ; \mathrm{H}$, 4.66; N, 6.47; S, 29.63\%; M, 859.84. $\Delta_{\mathrm{m}}$ at $25^{\circ} \mathrm{C}$ $\left(\Omega^{-1} \mathrm{~cm}^{2} \mathrm{~mol}^{-1}\right): 13.2$ in $\mathrm{MeOH}$. Selected infrared absorption $\left(\mathrm{KBr}, \mathrm{cm}^{-1}\right): v(\mathrm{CS}), 812,837 \mathrm{w} ; v(\mathrm{C}=\mathrm{S}), 933$, $987 \mathrm{~s} ; \quad v(\mathrm{CN}), 1,440,1,472,1,535 \mathrm{~s} .{ }^{1} \mathrm{H}-\mathrm{NMR}$ Spectra (300 MHz, $\delta$, DMSO): $\delta\left(\mathrm{CH}_{3}\right), 2.824-2.278$ (t, $\left.\left.24 \mathrm{H}\right) ;\right) ; \delta$ $\left(\mathrm{CH}_{2}\right), \quad 1.378-1.181$ (qrt, $\left.16 \mathrm{H}\right) .{ }^{13} \mathrm{C}-\mathrm{NMR}$ Spectra (300 MHz, $\delta$, DMSO): $\delta\left(\mathrm{CH}_{3}\right), 13.84 ; \delta\left(\mathrm{CH}_{2}\right), 51.19 ; \delta$ $(\mathrm{C}=\mathrm{S}), \quad$ 199.06. $\quad$ FAB-mass $\mathrm{m} / \mathrm{z}: \quad[\mathrm{ZnCl}]^{+}=100.8$; $\left[\mathrm{C}_{4} \mathrm{H}_{10} \mathrm{ClNZn}\right]^{+}=170.98 ; \quad\left[\mathrm{C}_{5} \mathrm{H}_{10} \mathrm{ClNSZn}\right]^{+}=214.95$; $\left[\mathrm{C}_{5} \mathrm{H}_{10} \mathrm{ClNS}_{2} \mathrm{Zn}\right]^{+}=246.92 ; \quad\left[\mathrm{C}_{5} \mathrm{H}_{10} \mathrm{ClNS}_{3} \mathrm{Zn}\right]^{+}=278.9$; $\left[\mathrm{C}_{6} \mathrm{H}_{10} \mathrm{ClNS}_{4} \mathrm{Zn}\right]^{+}=322.87 ;\left[\mathrm{C}_{4} \mathrm{H}_{10} \mathrm{Cl}_{3} \mathrm{NZn}_{2}\right]^{+}=304.85$; $\left[\mathrm{C}_{6} \mathrm{H}_{10} \mathrm{ClN}_{2} \mathrm{~S} 4 \mathrm{Zn}\right]^{+}=336.87 ;\left[\mathrm{C}_{5} \mathrm{H}_{10} \mathrm{Cl}_{3} \mathrm{NSZn}_{2}\right]^{+}=348.82$; $\left[\mathrm{C}_{5} \mathrm{H}_{10} \mathrm{Cl}_{3} \mathrm{NS}_{2} \mathrm{Zn}_{2}\right]^{+}=380.79 ;\left[\mathrm{C}_{10} \mathrm{H}_{20} \mathrm{ClN}_{2} \mathrm{~S}_{4} \mathrm{Zn}\right]^{+}=394.95$; $\left[\mathrm{C}_{5} \mathrm{H}_{10} \mathrm{Cl}_{3} \mathrm{NS}_{3} \mathrm{Zn}_{2}\right]^{+}=412.76 ;\left[\mathrm{C}_{6} \mathrm{H}_{10} \mathrm{Cl}_{3} \mathrm{NS}_{4} \mathrm{Zn}_{2}\right]^{+}=456.73$; $\left[\mathrm{C}_{14} \mathrm{H}_{30} \mathrm{ClN}_{3} \mathrm{~S} \mathrm{Z}_{4}\right]^{+}=467.03 ;\left[\mathrm{C}_{15} \mathrm{H}_{30} \mathrm{ClN}_{3} \mathrm{~S}_{5} \mathrm{Zn}\right]^{+}=511.00$; $\left[\mathrm{C}_{10} \mathrm{H}_{20} \mathrm{Cl}_{3} \mathrm{~N}_{2} \mathrm{~S}_{4} \mathrm{Zn}_{2}\right]^{+}=528.82 ; \quad\left[\mathrm{C}_{15} \mathrm{H}_{30} \mathrm{ClN}_{3} \mathrm{~S}_{6} \mathrm{Zn}\right]^{+}=$ 542.97; $\left.\left[\mathrm{C}_{15} \mathrm{H}_{30} \mathrm{ClN}_{3} \mathrm{~S}_{7} \mathrm{Zn}\right]^{+}=574.95 ; \mathrm{C}_{16} \mathrm{H}_{30} \mathrm{ClN}_{3} \mathrm{~S}_{8} \mathrm{Zn}\right]^{+}=$ 618.92; $\quad\left[\mathrm{C}_{15} \mathrm{H}_{30} \mathrm{Cl}_{3} \mathrm{~N}_{3} \mathrm{~S}_{5} \mathrm{Zn}_{2}\right]^{+}=644.87 ; \quad\left[\mathrm{C}_{15} \mathrm{H}_{30} \mathrm{Cl}_{3} \mathrm{~N}_{3} \mathrm{~S}_{6} \mathrm{Z}\right.$ $\left.\mathrm{n}_{2}\right]^{+}=674.84 ;\left[\mathrm{C}_{20} \mathrm{H}_{40} \mathrm{ClN}_{4} \mathrm{~S}_{8} \mathrm{Zn}\right]^{+}=691.00 ;\left[\mathrm{C}_{15} \mathrm{H}_{30} \mathrm{Cl}_{3} \mathrm{~N}_{3}\right.$ $\left.\mathrm{S}_{7} \mathrm{Zn}_{2}\right]^{+}=708.81 ; \quad\left[\mathrm{C}_{16} \mathrm{H}_{30} \mathrm{Cl}_{3} \mathrm{~N}_{3} \mathrm{~S}_{8} \mathrm{Zn}_{2}\right]^{+}=752.79 ; \quad\left[\mathrm{C}_{20} \mathrm{H}_{40}\right.$ $\left.\mathrm{Cl}_{3} \mathrm{~N}_{4} \mathrm{~S}_{8} \mathrm{Zn}_{2}\right]^{+}=824.87 ; \quad\left[\mathrm{C}_{20} \mathrm{H}_{40} \mathrm{Cl}_{4} \mathrm{~N}_{4} \mathrm{~S}_{8} \mathrm{Zn}_{2}+\mathrm{H}^{+}\right]^{+}=$ 860.84 .

\section{Synthesis of $\left[\mathrm{Zn}(\mathrm{TETD})_{4}\right] \mathrm{Cl}_{2}$, complex 2}

Zinc chloride $(0.136 \mathrm{~g}, 0.001 \mathrm{~mol})$ was dissolved in $20 \mathrm{~mL}$ of ethanol in a two-neck flask. The ligand TETD, (1.186 g, $0.004 \mathrm{~mol}$ ), dissolved in $100 \mathrm{~mL}$ of ethanol, was added to the above solution and stirred for 2-3 h. A dark-coloured solution was obtained after refluxing 15-20 h, which was evaporated and a dark grey-coloured precipitate was obtained, which was dried at room temperature and recrystallized from ethanol:acetone:chloroform 1:2:3 (v/v) solvent mixture. Yield, (1.088 g, 82.31\%), mp $140-145{ }^{\circ} \mathrm{C}$. found: $\mathrm{C}, 36.31 ; \mathrm{H}, 6.01 ; \mathrm{N}, 8.42 ; \mathrm{S}$, $38.73 \% . \mathrm{C}_{40} \mathrm{H}_{80} \mathrm{~N}_{8} \mathrm{~S}_{16} \mathrm{ZnCl}_{2}$ requires: $\mathrm{C}, 36.33 ; \mathrm{H}, 6.09 ; \mathrm{N}$, 8.47; S, 38.79\%; M, 1,322.45. $\Delta_{\mathrm{m}}$ at $25{ }^{\circ} \mathrm{C}$ $\left(\Omega^{-1} \mathrm{~cm}^{2} \mathrm{~mol}^{-1}\right)$ : 171.2 in DMSO. Selected infrared absorption $\left(\mathrm{KBr}, \mathrm{cm}^{-1}\right)$ : $v(\mathrm{CS}), 810,862 \mathrm{w} ; v(\mathrm{C}=\mathrm{S}), 937$, 978s; $v(\mathrm{CN}), 1,448,1,488 \mathrm{~s} .{ }^{1} \mathrm{H}-\mathrm{NMR}$ Spectra $(300 \mathrm{MHz}$, $\delta$, DMSO): $\delta\left(\mathrm{CH}_{3}\right), 2.849-2.798(\mathrm{~m}, 32 \mathrm{H}) ; \delta(\mathrm{C}-\mathrm{H})$, 1.029-1.242 (m, 48H). ${ }^{13} \mathrm{C}-\mathrm{NMR}$ Spectra $(300 \mathrm{MHz}, \delta$, DMSO): $\delta\left(\mathrm{CH}_{3}\right), 11.08 ; \delta\left(\mathrm{CH}_{2}\right), 51.33 ; \delta(\mathrm{C}=\mathrm{S}), 199.78$. FAB-mass $\quad m / z: \quad\left[\mathrm{C}_{4} \mathrm{H}_{10} \mathrm{NZn}\right]^{2+}=136.01$; $\left[\mathrm{C}_{5} \mathrm{H}_{10} \mathrm{NSZn}\right]^{2+}=178.98 ; \quad\left[\mathrm{C}_{5} \mathrm{H}_{10} \mathrm{NS}_{2} \mathrm{Zn}\right]^{2+}=211.95$; $\left[\mathrm{C}_{5} \mathrm{H}_{10} \mathrm{NS}_{3} \mathrm{Zn}\right]^{2+}=243.93 ; \quad\left[\mathrm{C}_{6} \mathrm{H}_{10} \mathrm{NS}_{4} \mathrm{Zn}\right]^{2+}=287.9$; $\left[\mathrm{C}_{6} \mathrm{H}_{10} \mathrm{~N}_{2} \mathrm{~S}_{4} \mathrm{Zn}\right]^{2+}=301.9 ;\left[\mathrm{C}_{10} \mathrm{H}_{20} \mathrm{~N}_{2} \mathrm{~S}_{4} \mathrm{Zn}\right]^{2+}=359.98$; $\left[\mathrm{C}_{14} \mathrm{H}_{30} \mathrm{~N}_{2} \mathrm{~S}_{4} \mathrm{Zn}\right]^{2+}=418.06 ;\left[\mathrm{C}_{14} \mathrm{H}_{30} \mathrm{~N}_{3} \mathrm{~S}_{4} \mathrm{Zn}\right]^{2+}=432.06$; $\left[\mathrm{C}_{15} \mathrm{H}_{30} \mathrm{~N}_{3} \mathrm{~S}_{5} \mathrm{Zn}\right]^{2+}=476.03 ;\left[\mathrm{C}_{15} \mathrm{H}_{30} \mathrm{~N}_{3} \mathrm{~S}_{6} \mathrm{Zn}\right]^{2+}=508.01$; $\left[\mathrm{C}_{15} \mathrm{H}_{30} \mathrm{~N}_{3} \mathrm{~S}_{7} \mathrm{Zn}\right]^{2+}=539.98 ;\left[\mathrm{C}_{16} \mathrm{H}_{30} \mathrm{~N}_{3} \mathrm{~S}_{8} \mathrm{Zn}\right]^{2+}=583.95$; $\left[\mathrm{C}_{16} \mathrm{H}_{30} \mathrm{~N}_{4} \mathrm{~S}_{8} \mathrm{Zn}\right]^{2+}=597.95 ;\left[\mathrm{C}_{20} \mathrm{H}_{40} \mathrm{~N}_{4} \mathrm{~S}_{8} \mathrm{Zn}\right]^{2+}=656.03$; $\left[\mathrm{C}_{24} \mathrm{H}_{50} \mathrm{~N}_{4} \mathrm{~S}_{8} \mathrm{Zn}\right]^{2+}=714.11 ;\left[\mathrm{C}_{24} \mathrm{H}_{50} \mathrm{~N}_{5} \mathrm{~S}_{8} \mathrm{Zn}\right]^{2+}=728.11$;
$\left[\mathrm{C}_{25} \mathrm{H}_{50} \mathrm{~N}_{5} \mathrm{~S}_{9} \mathrm{Zn}\right]^{2+}=772.08 ;\left[\mathrm{C}_{25} \mathrm{H}_{50} \mathrm{~N}_{5} \mathrm{~S}_{10} \mathrm{Zn}\right]^{2+}=804.06$; $\left.\mathrm{C}_{25} \mathrm{H}_{50} \mathrm{~N}_{5} \mathrm{~S}_{11} \mathrm{Zn}\right]^{2+}=836.03 ;\left[\mathrm{C}_{26} \mathrm{H}_{50} \mathrm{~N}_{5} \mathrm{~S}_{12} \mathrm{Zn}\right]^{2+}=880.00$; $\left[\mathrm{C}_{26} \mathrm{H}_{50} \mathrm{~N}_{6} \mathrm{~S}_{12} \mathrm{Zn}\right]^{2+}=894.00 ;\left[\mathrm{C}_{30} \mathrm{H}_{60} \mathrm{~N}_{6} \mathrm{~S}_{12} \mathrm{Zn}\right]^{2+}=952.08$; $\left[\mathrm{C}_{34} \mathrm{H}_{70} \mathrm{~N}_{6} \mathrm{~S}_{12} \mathrm{Zn}\right]^{2+}=1,010.16 ; \quad\left[\mathrm{C}_{34} \mathrm{H}_{70} \mathrm{~N}_{7} \mathrm{~S}_{12} \mathrm{Zn}\right]^{2+}=$ 1,024.16; $\left[\mathrm{C}_{35} \mathrm{H}_{70} \mathrm{~N}_{7} \mathrm{~S}_{13} \mathrm{Zn}\right]^{2+}=1,068.14 ; \quad\left[\mathrm{C}_{35} \mathrm{H}_{70} \mathrm{~N}_{7} \mathrm{~S}_{14} \mathrm{Z}\right.$ $\mathrm{n}]^{2+}=1,100.11 ; \quad\left[\mathrm{C}_{35} \mathrm{H}_{70} \mathrm{~N}_{7} \mathrm{~S}_{15} \mathrm{Zn}\right]^{2+}=1,132.08$; $\left[\mathrm{C}_{36} \mathrm{H}_{70} \mathrm{~N}_{7} \mathrm{~S}_{16} \mathrm{Zn}\right]^{2+}=1,176.05 ; \quad\left[\mathrm{C}_{36} \mathrm{H}_{70} \mathrm{~N}_{8} \mathrm{~S}_{16} \mathrm{Zn}\right]^{2+}=$ $1,190.05 ;\left[\mathrm{C}_{40} \mathrm{H}_{80} \mathrm{~N}_{8} \mathrm{~S}_{16} \mathrm{Zn}\right]^{2+}=1,248.13$.

\section{Synthesis of $\left[\mathrm{Cd}(\mathrm{TETD}) \mathrm{Cl}_{2}\right]_{2}$, complex 3}

Cadmium chloride $(0.367 \mathrm{~g}, 0.002 \mathrm{~mol})$ was dissolved in $30 \mathrm{~mL}$ of ethanol in a two-neck flask. The ligand TETD $(0.593 \mathrm{~g}, 0.002 \mathrm{~mol})$, dissolved in $30 \mathrm{~mL}$ of ethanol, was added to the above solution and stirred for $1-2 \mathrm{~h}$ in inert atmosphere. Thereafter, cadmium chloride $(0.173 \mathrm{~g}$, $0.0009 \mathrm{~mol} \mathrm{~mol}$ ) dissolved in $20 \mathrm{~mL}$ of ethanol, was added to above reaction mixture. A light cream-coloured solution obtained after a reflux of 18-20 h, was evaporated to yield a dirty white precipitate, which was further recrystallized from ethanol:acetone:chloroform, 1: 2: $2(\mathrm{v} / \mathrm{v})$ solvent mixture. Yield, (0.933 g, $97.29 \%), \mathrm{mp} 185-190{ }^{\circ} \mathrm{C}$. found: C, 25.00; $\mathrm{H}, 4.20 ; \mathrm{N}, 5.82 ; \mathrm{S}, 26.72 \% . \mathrm{C}_{20} \mathrm{H}_{40} \mathrm{Cl}_{4} \mathrm{~N}_{4} \mathrm{~S}_{8} \mathrm{Cd}_{2}$ requires: C, 25.03; H, 4.20; N, 5.83; S, 26.73\%; M, 959.71. $\Delta_{\mathrm{m}}$ at $25{ }^{\circ} \mathrm{C}\left(\Omega^{-1} \mathrm{~cm}^{2} \mathrm{~mol}^{-1}\right): 20.6$ in DMSO. Selected infrared absorption $\left(\mathrm{KBr}, \mathrm{cm}^{-1}\right): v(\mathrm{CS}), 805,832 \mathrm{w} ; v(\mathrm{C}=\mathrm{S}), 910$, 977s; $v(\mathrm{CN}), 1,433,1,479,1,539 \mathrm{~s} .{ }^{1} \mathrm{H}-\mathrm{NMR}$ Spectra (300 MHz, $\delta$, DMSO): $\delta\left(\mathrm{CH}_{3}\right), 2.764-2.306$ (t, $\left.\left.24 \mathrm{H}\right) ;\right) ; \delta$ $\left(\mathrm{CH}_{2}\right), 1.162-1.431$ (qrt, $\left.16 \mathrm{H}\right) .{ }^{13} \mathrm{C}-\mathrm{NMR}$ Spectra $(300 \mathrm{MH}$ $\mathrm{z}, \delta, \mathrm{DMSO}): \delta\left(\mathrm{CH}_{3}\right), 15.38 ; \delta\left(\mathrm{CH}_{2}\right), 51.10 ; \delta(\mathrm{C}=\mathrm{S})$, 200.22. FAB-mass $m / z: \quad\left[\mathrm{C}_{5} \mathrm{H}_{10} \mathrm{ClNCdS}\right]^{+}=264.93$; $\left[\mathrm{C}_{5} \mathrm{H}_{10} \mathrm{ClNCdS}_{2}\right]^{+}=296.9 ;\left[\mathrm{C}_{5} \mathrm{H}_{10} \mathrm{ClNS}_{3} \mathrm{Cd}\right]^{+}=328.87$; $\left[\mathrm{C}_{6} \mathrm{H}_{10} \mathrm{ClNS}_{4} \mathrm{Cd}\right]^{+}=372.84 ;\left[\mathrm{C}_{10} \mathrm{H}_{20} \mathrm{ClN}_{2} \mathrm{~S}_{4} \mathrm{Cd}\right]^{+}=444.92$; $\left[\mathrm{C}_{5} \mathrm{H}_{10} \mathrm{Cl}_{3} \mathrm{NCd}_{2} \mathrm{~S}\right]^{+}=448.77 ; \quad\left[\mathrm{C}_{5} \mathrm{H}_{10} \mathrm{Cl}_{3} \mathrm{NCd}_{2} \mathrm{~S}_{2}\right]^{+}=4$ 80.74; $\left[\mathrm{C}_{14} \mathrm{H}_{30} \mathrm{ClN}_{2} \mathrm{~S}_{4} \mathrm{Cd}\right]^{+}=503.00 ; \quad\left[\mathrm{C}_{5} \mathrm{H}_{10} \mathrm{Cl}_{3} \mathrm{NS}_{3} \mathrm{Cd}_{2}\right]^{+}$ $=512.71 ; \quad\left[\mathrm{C}_{6} \mathrm{H}_{10} \mathrm{Cl}_{3} \mathrm{NS}_{4} \mathrm{Cd}_{2}\right]^{+}=556.68 ; \quad\left[\mathrm{C}_{15} \mathrm{H}_{30} \mathrm{ClN}_{3} \mathrm{~S}_{5}\right.$ $\mathrm{Cd}]^{+}=560.98 ; \quad\left[\mathrm{C}_{15} \mathrm{H}_{30} \mathrm{ClN}_{3} \mathrm{~S}_{6} \mathrm{Cd}\right]^{+}=592.95 ;\left[\mathrm{C}_{15} \mathrm{H}_{30} \mathrm{Cl}\right.$ $\left.\mathrm{N}_{3} \mathrm{~S}_{7} \mathrm{Cd}\right]^{+}=624.92 ;\left[\mathrm{C}_{10} \mathrm{H}_{20} \mathrm{Cl}_{3} \mathrm{~N}_{2} \mathrm{~S}_{4} \mathrm{Cd}_{2}\right]^{+}=628.76 ;\left[\mathrm{C}_{16}\right.$ $\left.\mathrm{H}_{30} \mathrm{ClN}_{3} \mathrm{~S}_{8} \mathrm{Cd}\right]^{+}=668.89 ; \quad\left[\mathrm{C}_{20} \mathrm{H}_{40} \mathrm{ClN}_{4} \mathrm{~S}_{8} \mathrm{Cd}\right]^{+}=740.97$; $\left[\mathrm{C}_{15} \mathrm{H}_{30} \mathrm{Cl}_{3} \mathrm{~N}_{3} \mathrm{~S}_{5} \mathrm{Cd}_{2}\right]^{+}=744.82 ; \quad\left[\mathrm{C}_{15} \mathrm{H}_{30} \mathrm{Cl}_{3} \mathrm{~N}_{3} \mathrm{~S}_{6} \mathrm{Cd}_{2}\right]^{+}=7$ 76.79; $\left[\mathrm{C}_{15} \mathrm{H}_{30} \mathrm{Cl}_{3} \mathrm{~N}_{3} \mathrm{~S}_{7} \mathrm{Cd}_{2}\right]^{+}=808.76 ;\left[\mathrm{C}_{20} \mathrm{H}_{40} \mathrm{Cl}_{3} \mathrm{~N}_{4} \mathrm{~S}_{8} \mathrm{Cd}_{2}\right]^{+}$ $=924.82 ;\left[\mathrm{C}_{20} \mathrm{H}_{40} \mathrm{Cl}_{4} \mathrm{~N}_{4} \mathrm{~S}_{8} \mathrm{Cd}_{2}+\mathrm{H}^{+}\right]^{+}=960.78$.

\section{Synthesis of $\left[C d(T E T D)_{4}\right] C l_{2}$, complex 4}

Cadmium chloride $(0.183 \mathrm{~g}, 0.001 \mathrm{~mol})$ was dissolved in $30 \mathrm{~mL}$ of ethanol in a two-neck flask. The ligand TETD (1.186 g, $0.004 \mathrm{~mol})$, dissolved in $50 \mathrm{~mL}$ of ethanol, was added to the above solution and stirred for 2-3 h. A darkcoloured solution was obtained after refluxing 15-20 h, which was evaporated under vacuum to yield light greycoloured precipitate. The solid was recrystallized from ethanol:acetone:chloroform, 1:2:4 (v/v) solvent mixture. 
Yield $\left(1.210 \mathrm{~g}, 88.39 \%\right.$ ), $\mathrm{mp} 205-210{ }^{\circ} \mathrm{C}$. found: C, $35.01 ; \mathrm{H}, 5.72 ; \mathrm{N}, 8.13 ; \mathrm{S}, 37.42 \% . \mathrm{C}_{40} \mathrm{H}_{80} \mathrm{Cl}_{2} \mathrm{~N}_{8} \mathrm{~S}_{16} \mathrm{Cd}$ requires: $\mathrm{C}, 35.08 ; \mathrm{H}, 5.89 ; \mathrm{N}, 8.18 ; \mathrm{S}, 37.46 \% ; \mathrm{M}$, 1,369.47. $\Delta_{\mathrm{m}}$ at $25{ }^{\circ} \mathrm{C}\left(\Omega^{-1} \mathrm{~cm}^{2} \mathrm{~mol}^{-1}\right) 165.5$ in $\mathrm{MeOH}$. Selected infrared absorption $\left(\mathrm{KBr}, \mathrm{cm}^{-1}\right): v(\mathrm{CS}), 814$, 856w; $v(\mathrm{C}=\mathrm{S}), 917,998 \mathrm{~s} ; v(\mathrm{CN}), 1,421,1,477 \mathrm{~s} .{ }^{1} \mathrm{H}-\mathrm{NMR}$ Spectra (300 MHz, $\delta$, DMSO): $\delta\left(\mathrm{CH}_{3}\right), 2.879-3.798(\mathrm{~m}$, $32 \mathrm{H}) ; \delta\left(\mathrm{CH}_{2}\right), 1.028-1.249(\mathrm{~m}, 48 \mathrm{H}) .{ }^{13} \mathrm{C}-\mathrm{NMR}$ Spectra (300 MHz, $\delta$, DMSO): $\delta\left(\mathrm{CH}_{3}\right), 14.21 ; \delta\left(\mathrm{CH}_{2}\right), 51.27 ; \delta$ $(\mathrm{C}=\mathrm{S}), 201.56$. FAB-mass $\mathrm{m} / \mathrm{z}:\left[\mathrm{C}_{4} \mathrm{H}_{10} \mathrm{NCd}\right]^{2+}=185.98$; $\left[\mathrm{C}_{5} \mathrm{H}_{10} \mathrm{NSCd}\right]^{2+}=229.96 ; \quad\left[\mathrm{C}_{5} \mathrm{H}_{10} \mathrm{NS}_{2} \mathrm{Cd}\right]^{2+}=261.93$; $\left[\mathrm{C}_{5} \mathrm{H}_{10} \mathrm{NS}_{3} \mathrm{Cd}\right]^{2+}=293.9 ; \quad\left[\mathrm{C}_{6} \mathrm{H}_{10} \mathrm{NS}_{4} \mathrm{Cd}\right]^{2+}=337.87$; $\left[\mathrm{C}_{6} \mathrm{H}_{10} \mathrm{~N}_{2} \mathrm{~S}_{4} \mathrm{Cd}\right]^{2+}=351.88 ;\left[\mathrm{C}_{10} \mathrm{H}_{20} \mathrm{~N}_{2} \mathrm{~S}_{4} \mathrm{Cd}\right]^{2+}=409.95$; $\left[\mathrm{C}_{14} \mathrm{H}_{30} \mathrm{~N}_{2} \mathrm{~S}_{4} \mathrm{Cd}\right]^{2+}=468.03 ;\left[\mathrm{C}_{14} \mathrm{H}_{30} \mathrm{~N}_{3} \mathrm{~S}_{4} \mathrm{Cd}\right]^{2+}=482.04$; $\left[\mathrm{C}_{15} \mathrm{H}_{30} \mathrm{~N}_{3} \mathrm{~S}_{5} \mathrm{Cd}\right]^{2+}=526.01 ;\left[\mathrm{C}_{15} \mathrm{H}_{30} \mathrm{~N}_{3} \mathrm{~S}_{6} \mathrm{Cd}\right]^{2+}=557.98$; $\left[\mathrm{C}_{15} \mathrm{H}_{30} \mathrm{~N}_{3} \mathrm{~S}_{7} \mathrm{Cd}\right]^{2+}=589.95 ;\left[\mathrm{C}_{16} \mathrm{H}_{30} \mathrm{~N}_{3} \mathrm{~S}_{8} \mathrm{Cd}\right]^{2+}=633.92$; $\left[\mathrm{C}_{16} \mathrm{H}_{30} \mathrm{~N}_{4} \mathrm{~S}_{8} \mathrm{Cd}\right]^{2+}=647.93 ;\left[\mathrm{C}_{20} \mathrm{H}_{40} \mathrm{~N}_{4} \mathrm{~S}_{8} \mathrm{Cd}\right]^{2+}=706.01$; $\left[\mathrm{C}_{24} \mathrm{H}_{50} \mathrm{~N}_{4} \mathrm{~S}_{8} \mathrm{Cd}\right]^{2+}=764.08 ;\left[\mathrm{C}_{24} \mathrm{H}_{50} \mathrm{~N}_{5} \mathrm{~S}_{8} \mathrm{Cd}\right]^{2+}=778.09$; $\left[\mathrm{C}_{25} \mathrm{H}_{50} \mathrm{~N}_{5} \mathrm{~S}_{9} \mathrm{Cd}\right]^{2+}=882.06 ;\left[\mathrm{C}_{25} \mathrm{H}_{50} \mathrm{~N}_{5} \mathrm{~S}_{10} \mathrm{Cd}\right]^{2+}=854.03$; $\left[\mathrm{C}_{25} \mathrm{H}_{50} \mathrm{~N}_{5} \mathrm{~S}_{11} \mathrm{Cd}\right]^{2+}=886.00 ;\left[\mathrm{C}_{26} \mathrm{H}_{50} \mathrm{~N}_{5} \mathrm{~S}_{12} \mathrm{Cd}\right]^{2+}=929.97$; $\left[\mathrm{C}_{26} \mathrm{H}_{50} \mathrm{~N}_{6} \mathrm{~S}_{12} \mathrm{Cd}\right]^{2+}=943.98 ; \quad\left[\mathrm{C}_{30} \mathrm{H}_{60} \mathrm{~N}_{6} \mathrm{~S}_{12} \mathrm{Cd}\right]^{2+}=$ 1,002.06; $\left[\mathrm{C}_{34} \mathrm{H}_{70} \mathrm{~N}_{6} \mathrm{~S}_{12} \mathrm{Cd}\right]^{2+}=1,060.13 ;\left[\mathrm{C}_{34} \mathrm{H}_{70} \mathrm{~N}_{7} \mathrm{~S}_{12} \mathrm{C}\right.$ d $]^{2+}=1,074.14 ;\left[\mathrm{C}_{35} \mathrm{H}_{70} \mathrm{~N}_{7} \mathrm{~S}_{13} \mathrm{Cd}\right]^{2+}=1,118.11 ;\left[\mathrm{C}_{35} \mathrm{H}_{70}\right.$ $\left.\mathrm{N}_{7} \mathrm{~S}_{14} \mathrm{Cd}\right]^{2+}=1,150.08 ; \quad\left[\mathrm{C}_{35} \mathrm{H}_{70} \mathrm{~N}_{7} \mathrm{~S}_{15} \mathrm{Cd}\right]^{2+}=1,182.05$; $\left[\mathrm{C}_{36} \mathrm{H}_{70} \mathrm{~N}_{7} \mathrm{~S}_{16} \mathrm{Cd}\right]^{2+}=1,226.03 ; \quad\left[\mathrm{C}_{36} \mathrm{H}_{70} \mathrm{~N}_{8} \mathrm{~S}_{16} \mathrm{Cd}\right]^{2+}=$ $1,240.03 ;\left[\mathrm{C}_{40} \mathrm{H}_{80} \mathrm{~N}_{8} \mathrm{~S}_{16} \mathrm{Cd}\right]^{2+}=1,298.11$.

\section{Synthesis of $\left[\mathrm{Hg}(\mathrm{TETD}) \mathrm{Cl}_{2}\right]_{2}$, complex 5}

Mercury chloride $(0.543 \mathrm{~g}, 0.002 \mathrm{~mol})$ was dissolved in $30 \mathrm{~mL}$ of ethanol in a two-neck flask. The ligand TETD $(0.593 \mathrm{~g}, 0.002 \mathrm{~mol})$ dissolved in $30 \mathrm{~mL}$ of ethanol was added to the above solution and stirred for $2-3 \mathrm{~h}$. Thereafter, mercury chloride $(0.136 \mathrm{~g}, 0.0005 \mathrm{~mol})$ dissolved in $20 \mathrm{~mL}$ ethanol, was added to above reaction mixture. A colourless solution was obtained after refluxing 18-20 h, which was evaporated under vacuum to yield light grey precipitate, which was dried at room temperature and recrystallized from ethanol:acetone:chloroform, 1:1:1 (v/v) solvent mixture. Yield $(0.956 \mathrm{~g}, 84.14 \%), \quad \mathrm{mp}$ $135-140{ }^{\circ} \mathrm{C}$. found: $\mathrm{C}, 21.11 ; \mathrm{H}, 3.55 ; \mathrm{N}, 4.92 ; \mathrm{S}, 22.56 \%$. $\mathrm{C}_{20} \mathrm{H}_{40} \mathrm{Cl}_{4} \mathrm{~N}_{4} \mathrm{~S}_{8} \mathrm{Hg}_{2}$ requires: $\mathrm{C}, 21.14 ; \mathrm{H}, 3.55 ; \mathrm{N}, 4.93 ; \mathrm{S}$, $22.58 \% ; \mathrm{M}, 1,136.07 . \Delta_{\mathrm{m}}$ at $25{ }^{\circ} \mathrm{C}\left(\Omega^{-1} \mathrm{~cm}^{2} \mathrm{~mol}^{-1}\right): 17.9$ in DMSO. Selected infrared absorption $\left(\mathrm{KBr}, \mathrm{cm}^{-1}\right): v$ (CS), 808, 839w; $v(\mathrm{C}=\mathrm{S}), 912,983 \mathrm{~s} ; v(\mathrm{CN}), 1,454,1,492$, 1,550s. ${ }^{1} \mathrm{H}-\mathrm{NMR}$ Spectra (300 MHz, $\delta$, DMSO): $\delta\left(\mathrm{CH}_{3}\right)$, $2.949-2.720$ (t, $24 \mathrm{H}) ;) ; \delta\left(\mathrm{CH}_{2}\right), 1.492-1.196$ (qrt, 16H). ${ }^{13} \mathrm{C}$-NMR Spectra (300 MHz, $\delta$, DMSO): $\delta\left(\mathrm{CH}_{3}\right), 11.77 ; \delta$ $\left(\mathrm{CH}_{2}\right), \quad 51.06 ; \quad \delta \quad(\mathrm{C}=\mathrm{S}), \quad 201.27$. ESI-mass $\mathrm{m} / \mathrm{z}$ : $\mathrm{HgCl}]^{+}=236.7 ;\left[\mathrm{Hg}^{202} \mathrm{Cl}\right]^{+}=238.7 ;\left[\mathrm{C}_{4} \mathrm{H}_{10} \mathrm{ClNHg}\right]^{+}=$
309.02; $\left[\mathrm{C}_{5} \mathrm{H}_{10} \mathrm{ClNHg}^{202} \mathrm{~S}^{+}=355.0 ;\left[\mathrm{C}_{5} \mathrm{H}_{10} \mathrm{ClNHgS}_{2}\right]^{+}=\right.$ 384.96; $\left[\mathrm{C}_{6} \mathrm{H}_{10} \mathrm{ClNS}_{4} \mathrm{Hg}\right]^{+}=460.91 ;\left[\mathrm{C}_{6} \mathrm{H}_{10} \mathrm{ClNS}_{3} \cdot \mathrm{S}^{34} \mathrm{Hg}\right]^{+}$ $=462.91\left[\mathrm{C}_{10} \mathrm{H}_{20} \mathrm{ClN}_{2} \mathrm{~S}_{4} \mathrm{Hg}\right]^{+}=532.99 ;\left[\mathrm{C}_{14} \mathrm{H}_{30} \mathrm{ClN}_{2} \mathrm{~S}_{4} \mathrm{H}\right.$ $\mathrm{g}]^{+}=591.07 ;\left[\mathrm{C}_{14} \mathrm{H}_{30} \mathrm{ClN}_{3} \mathrm{~S}_{4} \mathrm{Hg}\right]^{+}=604.90 ;\left[\mathrm{C}_{14} \mathrm{H}_{30} \mathrm{ClN}_{2}\right.$. $\left.\mathrm{N}^{15} \mathrm{~S}_{4} \mathrm{Hg}\right]^{+}=605.90 \quad\left[\mathrm{C}_{5} \mathrm{H}_{10} \mathrm{Cl}_{3} \mathrm{NHg}_{2} \mathrm{~S}_{2}\right]^{+}=656.87 ; \quad\left[\mathrm{C}_{12}\right.$ $\left.\mathrm{H}_{20} \mathrm{ClN}_{2} \mathrm{~S}_{8} \mathrm{Hg}\right]^{+}=684.0 ;\left[\mathrm{C}_{12} \mathrm{H}_{20} \mathrm{ClN} . \mathrm{N}^{15} \mathrm{~S}_{8} \mathrm{Hg}\right]^{+}=685.0$ $\left[\mathrm{C}_{16} \mathrm{H}_{30} \mathrm{ClN}_{3} \mathrm{~S}_{8} \mathrm{Hg}^{202}\right]^{+}=758.0 ;\left[\mathrm{C}_{20} \mathrm{H}_{40} \mathrm{ClN}_{4} \mathrm{~S}_{8} \mathrm{Hg}\right]^{+}=82$ 9.04; $\left[\mathrm{C}_{20} \mathrm{H}_{40} \mathrm{Cl}_{2} \mathrm{~N}_{4} \mathrm{~S}_{8} \mathrm{Hg}\right]^{+}=864.01 ;\left[\mathrm{C}_{20} \mathrm{H}_{40} \mathrm{Cl}_{2} \mathrm{~N}_{3} \cdot \mathrm{N}^{15} \mathrm{~S}_{8}\right.$ $\mathrm{Hg}]=865.01 .\left[\mathrm{C}_{15} \mathrm{H}_{30} \mathrm{Cl}_{3} \mathrm{~N}_{3} \mathrm{Hg}_{2} \mathrm{~S}_{6}\right]^{+}=952.92 ;\left[\mathrm{C}_{20} \mathrm{H}_{40} \mathrm{Cl}_{3}\right.$ $\left.\mathrm{N}_{4} \mathrm{~S}_{8} \mathrm{Hg}_{2}\right]^{+}=1,100.95 ;\left[\mathrm{C}_{20} \mathrm{H}_{40} \mathrm{Cl}_{4} \mathrm{~N}_{4} \mathrm{~S}_{8} \mathrm{Hg}_{2}\right]^{+}=1,135.92$. $\mathrm{C}_{20} \mathrm{H}_{40} \mathrm{Cl}_{4} \mathrm{~N}_{4} \mathrm{~S}_{8} \mathrm{Hg}_{2}(\mathrm{M} \tau=1,136.07)$, requires: $\mathrm{C}, 21.14 ; \mathrm{H}$, $3.55 ; \mathrm{N}, 4.93 ; \mathrm{S}, 22.58$. Found: C, 21.11; H, 3.55; N, 4.92; S, 22.56 .

\section{Synthesis of $\left[\mathrm{Hg}(\mathrm{TETD})_{4}\right] \mathrm{Cl}_{2}$, complex 6}

Mercury chloride $(0.272 \mathrm{~g}, 0.001 \mathrm{~mol})$ was dissolved in $50 \mathrm{~mL}$ of ethanol in a two-neck flask. The ligand TETD $(1.186 \mathrm{~g}, 0.004 \mathrm{~mol})$, dissolved in $100 \mathrm{~mL}$ of ethanol, was added to the above solution and stirred for $2-3 \mathrm{~h}$. A colourless solution was obtained after refluxing 18-20 h, which was evaporated and greyish precipitate was obtained, which was dried at room temperature and recrystallized from ethanol:acetone:chloroform, 1:2:2 (v/v) solvent mixture. Yield $(1.345 \mathrm{~g}, 92.25 \%), \mathrm{mp} 85-90{ }^{\circ} \mathrm{C}$. Found: C, $32.91 ; \mathrm{H}, 5.50 ; \mathrm{N}, 7.62 ; \mathrm{S}, 35.13 \% . \mathrm{C}_{40} \mathrm{H}_{80} \mathrm{Cl}_{2} \mathrm{~N}_{8} \mathrm{~S}_{16} \mathrm{Hg}$ requires: $\mathrm{C}, 32.95 ; \mathrm{H}, 5.53 ; \mathrm{N}, 7.69 ; \mathrm{S}, 35.20 \% ; \mathrm{M}$, 1,457.65. $\Delta_{\mathrm{m}}$ at $25{ }^{\circ} \mathrm{C}\left(\Omega^{-1} \mathrm{~cm}^{2} \mathrm{~mol}^{-1}\right): 159.6$ in DMSO. Selected infrared absorption $\left(\mathrm{KBr}, \mathrm{cm}^{-1}\right): v(\mathrm{CS}), 817$, $847 \mathrm{w} ; v(\mathrm{C}=\mathrm{S}), 908,972 \mathrm{~s} ; v(\mathrm{CN}), 1,446,1,483 \mathrm{~s} .{ }^{1} \mathrm{H}-\mathrm{NMR}$ Spectra $\left(300 \mathrm{MHz}, \delta\right.$, DMSO): $\delta\left(\mathrm{CH}_{3}\right), 2.938-2.833(\mathrm{t}$, $32 \mathrm{H}) ; \delta\left(\mathrm{CH}_{2}\right), 1.251-1.036(\mathrm{qrt}, 32 \mathrm{H}){ }^{13} \mathrm{C}-\mathrm{NMR}$ Spectra (300 MHz, $\delta$, DMSO): $\delta\left(\mathrm{CH}_{3}\right), 10.82 ; \delta\left(\mathrm{CH}_{2}\right), 51.48 ; \delta$ $(\mathrm{C}=\mathrm{S}), 203.97$. FAB-mass $\mathrm{m} / \mathrm{z}:\left[\mathrm{C}_{4} \mathrm{H}_{10} \mathrm{NHg}\right]^{2+}=274.05$; $\left[\mathrm{C}_{5} \mathrm{H}_{10} \mathrm{NSHg}\right]^{2+}=318.02 ; \quad\left[\mathrm{C}_{5} \mathrm{H}_{10} \mathrm{NS}_{2} \mathrm{Hg}\right]^{2+}=350.00 ;$ $\left[\mathrm{C}_{5} \mathrm{H}_{10} \mathrm{NS}_{3} \mathrm{Hg}\right]^{2+}=381.97 ; \quad\left[\mathrm{C}_{6} \mathrm{H}_{10} \mathrm{NS}_{4} \mathrm{Hg}\right]^{2+}=425.94 ;$ $\left[\mathrm{C}_{6} \mathrm{H}_{10} \mathrm{~N}_{2} \mathrm{~S}_{4} \mathrm{Hg}\right]^{2+}=439.94 ;\left[\mathrm{C}_{10} \mathrm{H}_{20} \mathrm{~N}_{2} \mathrm{~S}_{4} \mathrm{Hg}\right]^{2+}=498.02$; $\left[\mathrm{C}_{14} \mathrm{H}_{30} \mathrm{~N}_{2} \mathrm{~S}_{4} \mathrm{Hg}\right]^{2+}=556.10 ;\left[\mathrm{C}_{14} \mathrm{H}_{30} \mathrm{~N}_{3} \mathrm{~S}_{4} \mathrm{Hg}\right]^{2+}=570.10$; $\left[\mathrm{C}_{15} \mathrm{H}_{30} \mathrm{~N}_{3} \mathrm{~S}_{5} \mathrm{Hg}\right]^{2+}=614.07 ;\left[\mathrm{C}_{15} \mathrm{H}_{30} \mathrm{~N}_{3} \mathrm{~S}_{6} \mathrm{Hg}\right]^{2+}=646.05$; $\left[\mathrm{C}_{15} \mathrm{H}_{30} \mathrm{~N}_{3} \mathrm{~S}_{7} \mathrm{Hg}\right]^{2+}=678.02 ;\left[\mathrm{C}_{16} \mathrm{H}_{30} \mathrm{~N}_{3} \mathrm{~S}_{8} \mathrm{Hg}\right]^{2+}=721.99$; $\left[\mathrm{C}_{16} \mathrm{H}_{30} \mathrm{~N}_{4} \mathrm{~S}_{8} \mathrm{Hg}\right]^{2+}=735.99 ;\left[\mathrm{C}_{20} \mathrm{H}_{40} \mathrm{~N}_{4} \mathrm{~S}_{8} \mathrm{Hg}\right]^{2+}=794.07$; $\left[\mathrm{C}_{24} \mathrm{H}_{50} \mathrm{~N}_{4} \mathrm{~S}_{8} \mathrm{Hg}\right]^{2+}=852.15 ;\left[\mathrm{C}_{24} \mathrm{H}_{50} \mathrm{~N}_{5} \mathrm{~S}_{8} \mathrm{Hg}\right]^{2+}=866.15$; $\left[\mathrm{C}_{25} \mathrm{H}_{50} \mathrm{~N}_{5} \mathrm{~S}_{9} \mathrm{Hg}\right]^{2+}=910.13 ;\left[\mathrm{C}_{25} \mathrm{H}_{50} \mathrm{~N}_{5} \mathrm{~S}_{10} \mathrm{Hg}\right]^{2+}=942$. $10 ; \quad\left[\mathrm{C}_{25} \mathrm{H}_{50} \mathrm{~N}_{5} \mathrm{~S}_{11} \mathrm{Hg}\right]^{2+}=974.07 ; \quad\left[\mathrm{C}_{26} \mathrm{H}_{50} \mathrm{~N}_{5} \mathrm{~S}_{12} \mathrm{Hg}\right]^{2+}=$ 1,018.04; $\quad\left[\mathrm{C}_{26} \mathrm{H}_{50} \mathrm{~N}_{6} \mathrm{~S}_{12} \mathrm{Hg}\right]^{2+}=1,032.05 ; \quad\left[\mathrm{C}_{30} \mathrm{H}_{60}\right.$ $\left.\mathrm{N}_{6} \mathrm{~S}_{12} \mathrm{Hg}\right]^{2+}=1,090.12 ; \quad\left[\mathrm{C}_{34} \mathrm{H}_{70} \mathrm{~N}_{6} \mathrm{~S}_{12} \mathrm{Hg}\right]^{2+}=1,148.20$; $\left[\mathrm{C}_{34} \mathrm{H}_{70} \mathrm{~N}_{7} \mathrm{~S}_{12} \mathrm{Hg}\right]^{2+}=1,162.20 ; \quad\left[\mathrm{C}_{35} \mathrm{H}_{70} \mathrm{~N}_{7} \mathrm{~S}_{13} \mathrm{Hg}\right]^{2+}=$ 1,206.18; $\quad\left[\mathrm{C}_{35} \mathrm{H}_{70} \mathrm{~N}_{7} \mathrm{~S}_{14} \mathrm{Hg}\right]^{2+}=1,238.15 ; \quad\left[\mathrm{C}_{35} \mathrm{H}_{70} \mathrm{~N}_{7} \mathrm{~S}_{15}\right.$ $\mathrm{Hg}]^{2+}=1,270.12 ;\left[\mathrm{C}_{36} \mathrm{H}_{70} \mathrm{~N}_{7} \mathrm{~S}_{16} \mathrm{Hg}\right]^{2+}=1,314.09 ;\left[\mathrm{C}_{36} \mathrm{H}_{70}\right.$ $\left.\mathrm{N}_{8} \mathrm{~S}_{16} \mathrm{Hg}\right]^{2+}=1,328.10 ;\left[\mathrm{C}_{40} \mathrm{H}_{80} \mathrm{~N}_{8} \mathrm{~S}_{16} \mathrm{Hg}\right]^{2+}=1,386.17$. 
Synthesis of nanoparticles by thermal decomposition

\section{ZnS nanoparticles}

The synthesized complex [Zn(TETD) $\left.\mathrm{Cl}_{2}\right]_{2} /\left[\mathrm{Zn}(\mathrm{TETD})_{4}\right]$ $\mathrm{Cl}_{2}$, $(0.1 \mathrm{mmol})$ was mixed with triethanolamine $(0.027 \mathrm{~mL}, 0.2 \mathrm{mmol})$ and oleic acid $(0.063 \mathrm{~mL}$, $0.2 \mathrm{mmol}$ ) in $12 \mathrm{~mL}$ of toluene in a flat-bottom microflask equipped with a glass lid. Reaction mixture was mixed thoroughly and the closed microflask was kept in a muffle furnace at $\sim 200{ }^{\circ} \mathrm{C}$ for $1 \mathrm{~h}$. Thereafter, furnace was cooled till normal temperature was attained. A change in colour of reaction mixture was observed and a light brown precipitate was obtained. The insoluble precipitate was washed several times by toluene, followed by methanol and then centrifuged. It was vacuum dried at room temperature. The experiment was performed at different precursor:surfactant ratio at different temperature (Table 1).

\section{CdS nanoparticles}

The synthesized complex $\left[\mathrm{Cd}(\mathrm{TETD}) \mathrm{Cl}_{2}\right]_{2} /\left[\mathrm{Cd}(\mathrm{TETD})_{4}\right]$ $\mathrm{Cl}_{2},(0.2 \mathrm{mmol})$ was mixed with hexadecylamine $(0.289 \mathrm{~g}$, $1.2 \mathrm{mmol}$ ) in $10 \mathrm{~mL}$ of toluene in a flat-bottom microflask. Reaction mixture was mixed thoroughly and the closed microflask was kept in a muffle furnace at $\sim 300{ }^{\circ} \mathrm{C}$ for $1 \mathrm{~h}$. Thereafter, furnace was cooled till normal temperature was attained. A change in colour was observed and a yellow precipitate was obtained. Insoluble precipitate was washed several times by toluene, followed by methanol and then centrifuged. It was vacuum dried at room temperature.
The experiment was performed at different precursor:surfactant ratio at different temperature (Table 1).

\section{HgS nanoparticles}

The synthesized complex, $\left.\left[\mathrm{Hg}(\mathrm{TETD}) \mathrm{Cl}_{2}\right]_{2} /[\mathrm{Hg} \text { (TETD })_{4}\right]$ $\mathrm{Cl}_{2}$, $(0.05 \mathrm{mmol})$ was mixed with tri-octylphosphine oxide $(0.39 \mathrm{~g}, 0.1 \mathrm{mmol})$ in $10 \mathrm{~mL}$ of toluene in a flat-bottom microflask. Reaction mixture was mixed thoroughly and heated in a muffle furnace at $\sim 200{ }^{\circ} \mathrm{C}$ for $1 \mathrm{~h}$ and cooled. After cooling, colour of the reaction mixture was changed and a black precipitate was obtained. Insoluble precipitate was washed by toluene followed by methanol and centrifuged, and then it was vacuum dried at room temperature. The experiment was performed at different precursor:surfactant ratio at different temperature (Table 1).

\section{Antibacterial screening}

All the metal complexes from 1 to $\mathbf{6}$, ligand TETD and the nanoparticles of $\mathrm{CdS}, \mathrm{ZnS}$ and $\mathrm{HgS}$ were screened for antibacterial activity against Gram-negative bacteria Escherichia coli, MTCC 1304 at different concentrations. Mueller-Hinton agar plates (MHA) were prepared and $50 \mu \mathrm{L}$ suspensions of $E$. coli containing $\sim 10^{5} \mathrm{CFU}$ (colony-forming unit) were applied to the plate by the welldiffusion method. The wells were made on the plates and they were filled with $50 \mu \mathrm{L}$ of sample solution of $0.02 \%$ concentration except in nanoparticles, where $0.02 \%$ dried samples were introduced as such in the well. The $0.02 \%$ solution of chloramphenicol was used for comparison. These plates were incubated at $37 \pm 1{ }^{\circ} \mathrm{C}$ for $24-48 \mathrm{~h}$ in
Table 1 Effect of precursor:surfactant ratio on the size of nanoparticles

\begin{tabular}{|c|c|c|c|}
\hline Complexes & Surfactant & $\begin{array}{l}\text { Ratio of } \\
\text { complex:surfactant }\end{array}$ & $\begin{array}{l}\text { Mean particle size of } \\
\text { nanoparticles from } \\
\text { complexes (nm) }\end{array}$ \\
\hline 1,2 & Triethanolamine & $1: 1$ & $15.5 ; 15.90$ \\
\hline 1,2 & Triethanolamine & $1: 2$ & $8.95 ; 8.99$ \\
\hline 1,2 & Triethanolamine & $1: 3$ & $8.90 ; 8.94$ \\
\hline 1,2 & Triethanolamine & $1: 4$ & $8.94 ; 8.63$ \\
\hline 1,2 & Triethanolamine & $1: 5$ & $8.98 ; 8.79$ \\
\hline 3,4 & Hexadecylamine & $1: 1$ & $30.45 ; 30.87$ \\
\hline 3,4 & Hexadecylamine & $1: 2$ & $26.57 ; 26.46$ \\
\hline 3,4 & Hexadecylamine & $1: 3$ & $26.26 ; 26.12$ \\
\hline 3,4 & Hexadecylamine & $1: 4$ & $26.56 ; 26.37$ \\
\hline 3,4 & Hexadecylamine & $1: 5$ & $26.60 ; 26.72$ \\
\hline 5,6 & Tri-octylphosphine oxide & $1: 1$ & $13.45 ; 13.89$ \\
\hline 5,6 & Tri-octylphosphine oxide & $1: 2$ & $9.85 ; 9.2$ \\
\hline 5,6 & Tri-octylphosphine oxide & $1: 3$ & $9.56 ; 9.82$ \\
\hline 5,6 & Tri-octylphosphine oxide & $1: 4$ & $9.89 ; 9.70$ \\
\hline 5,6 & Tri-octylphosphine oxide & $1: 5$ & $9.90 ; 9.32$ \\
\hline
\end{tabular}


refrigerated incubator shakers. The results in the form of zone inhibition, measured in millimetres, are presented in Table 2.

Minimum inhibitory concentration (MIC)

To monitor the activity and confirm their sensitivity complexes from 1 to 6 and nanoparticles were tested for MIC. The most popular method for MIC evaluation, commonly used in clinical laboratory, is successive dilution method.

In six numbered screw tubes A-F $(10 \times 100 \mathrm{~mm})$, $1 \mathrm{~mL}$ of Mueller-Hinton broth medium was distributed in each tube, except for the tube $\mathbf{A}$. All the tubes were placed at autoclave for sterilization. In tubes $\mathbf{A}$ and $\mathbf{B}, 1 \mathrm{~mL}$ of test solution (1-6) was added; tube $\mathbf{B}$ was stirred and $1 \mathrm{~mL}$ of mixture of the same was taken out and transferred to tube C. The $0.1 \mathrm{~mL}$ suspension of inoculums E. coli, MTCC 1304 having $\sim 500,000$ cells per $\mu \mathrm{L}$ was added to all tubes. Incubation at $37 \pm 1{ }^{\circ} \mathrm{C}$ temperature was maintained for 24-48 h. MIC is concentration of the higher dilution tube, in which bacterial growth was absent and the results are presented in Table 2 .

\section{Results and discussion}

Six complexes of $\mathrm{Zn}(\mathrm{II}), \mathrm{Cd}(\mathrm{II})$ and $\mathrm{Hg}$ (II) were prepared according to the following equations:
Table 2 Antibacterial screening and MIC against E. coli

\begin{tabular}{|c|c|c|c|c|}
\hline S. no. & $\begin{array}{l}\text { Complex/ligand/ } \\
\text { nanoparticle }\end{array}$ & $\begin{array}{l}\text { Activity } \\
\text { against } \\
E \text {. Coli }\end{array}$ & $\begin{array}{l}\text { Inhibition } \\
\text { zone in } \\
\mathrm{mm}^{\mathrm{a}}\end{array}$ & $\begin{array}{l}\text { Minimum } \\
\text { inhibitory } \\
\text { concentration } \\
(\mu \mathrm{g} / \mathrm{mL})\end{array}$ \\
\hline & TETD & + & 18 & 0.68 \\
\hline 1. & {$\left[\mathrm{ZnCl}_{2}(\mathrm{TETD})\right]_{2}$} & + & 20 & 0.39 \\
\hline 2. & {$\left[\mathrm{Zn}(\mathrm{TETD})_{4}\right] \mathrm{Cl}_{2}$} & + & 23 & 0.34 \\
\hline 3. & {$\left[\mathrm{CdCl}_{2}(\mathrm{TETD})\right]_{2}$} & + & 42 & 0.35 \\
\hline 4. & {$\left[\mathrm{Cd}(\mathrm{TETD})_{4}\right] \mathrm{Cl}_{2}$} & + & 34 & 0.31 \\
\hline 5. & {$\left[\mathrm{HgCl}_{2} \text { (TETD) }\right]_{2}$} & + & 34 & 0.25 \\
\hline 6. & {$\left[\mathrm{Hg}(\mathrm{TETD})_{4}\right] \mathrm{Cl}_{2}$} & + & 38 & 0.23 \\
\hline i. & ZnS nanoparticle & + & 17 & 0.28 \\
\hline ii. & CdS nanoparticle & + & 28 & 0.26 \\
\hline iii. & $\mathrm{HgS}$ nanoparticle & + & 26 & 0.20 \\
\hline C. & Chloramphenicol & + & 42 & - \\
\hline
\end{tabular}

${ }^{a}$ Diameter of inhibition zone $>8 \mathrm{~mm}$ is taken as active and is shown as + in the table

604.90, 605.90, 656.87, 684.0, 685.0 758.0, 829.04, 952.92, $1,100.95,1,135.92,1,136.90$ were attributed for $[\mathrm{HgCl}]^{+}$, $\left[\mathrm{Hg}^{202} \mathrm{Cl}\right]^{+},\left[\mathrm{C}_{4} \mathrm{H}_{10} \mathrm{ClNHg}\right]^{+},\left[\mathrm{C}_{5} \mathrm{H}_{10} \mathrm{ClNHg}^{202} \mathrm{~S}^{+},\left[\mathrm{C}_{5} \mathrm{H}_{10}\right.\right.$ $\left.\mathrm{ClNHgS}_{2}\right]^{+}, \quad\left[\mathrm{C}_{6} \mathrm{H}_{10} \mathrm{ClNS}_{4} \mathrm{Hg}\right]^{+}, \quad\left[\mathrm{C}_{6} \mathrm{H}_{10} \mathrm{ClNS}_{3} \cdot \mathrm{S}^{34} \mathrm{Hg}\right]^{+}$, $\left[\mathrm{C}_{10} \mathrm{H}_{20} \mathrm{ClN}_{2} \mathrm{~S}_{4} \mathrm{Hg}\right]^{+},\left[\mathrm{C}_{14} \mathrm{H}_{30} \mathrm{ClN}_{2} \mathrm{~S}_{4} \mathrm{Hg}\right]^{+},\left[\mathrm{C}_{14} \mathrm{H}_{30} \mathrm{ClN}_{3} \mathrm{~S}_{4}\right.$ $\mathrm{Hg}]^{+} \quad\left[\mathrm{C}_{14} \mathrm{H}_{30} \mathrm{ClN}_{2} \cdot \mathrm{N}^{15} \mathrm{~S}_{4} \mathrm{Hg}\right]^{+}, \quad\left[\mathrm{C}_{5} \mathrm{H}_{10} \mathrm{Cl}_{3} \mathrm{NHg}_{2} \mathrm{~S}_{2}\right]^{+}$, $\left[\mathrm{C}_{12} \mathrm{H}_{20} \mathrm{ClN}_{2} \mathrm{~S}_{8} \mathrm{Hg}\right]^{+},\left[\mathrm{C}_{12} \mathrm{H}_{20} \mathrm{ClN} . \mathrm{N}^{15} \mathrm{~S}_{8} \mathrm{Hg}\right]^{+},\left[\mathrm{C}_{16} \mathrm{H}_{30} \mathrm{ClN}_{3}\right.$ $\left.\mathrm{S}_{8} \mathrm{Hg}^{202}\right]^{+}, \quad\left[\mathrm{C}_{20} \mathrm{H}_{40} \mathrm{ClN}_{4} \mathrm{~S}_{8} \mathrm{Hg}\right]^{+}, \quad\left[\mathrm{C}_{15} \mathrm{H}_{30} \mathrm{Cl}_{3} \mathrm{~N}_{3} \mathrm{Hg}_{2} \mathrm{~S}_{6}\right]^{+}$,

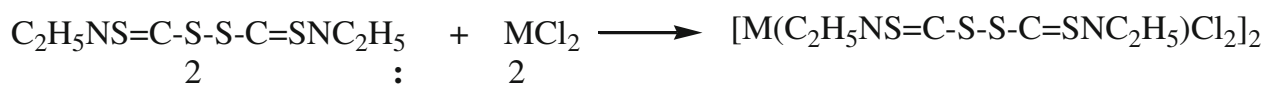

$\mathrm{C}_{2} \mathrm{H}_{5} \mathrm{NS}=\mathrm{C}-\mathrm{S}-\mathrm{S}-\mathrm{C}=\mathrm{SNC}_{2} \mathrm{H}_{5}+\mathrm{MCl}_{2} \longrightarrow\left[\mathrm{M}\left(\mathrm{C}_{2} \mathrm{H}_{5} \mathrm{NS}=\mathrm{C}-\mathrm{S}-\mathrm{S}-\mathrm{C}=\mathrm{SNC}_{2} \mathrm{H}_{5}\right] \mathrm{Cl}_{2}\right.$ $4 \quad: \quad 1$

where $\mathrm{M}=\mathrm{Zn}(\mathrm{II}), \mathrm{Cd}(\mathrm{II})$ and $\mathrm{Hg}(\mathrm{II})$.

Characterization of the complexes

Empirical formula of all the complexes 1-6 was in conformity with the elemental analysis. Molecular weights of all the complexes were confirmed by FAB-MS. A number of peaks were observed in mass spectra. Some important peaks, which were spotted in the FAB-MS of 2:2 complexes, exhibit $[\mathrm{MCl}]^{+}, \quad\left[\mathrm{C}_{20} \mathrm{H}_{40} \mathrm{ClN}_{4} \mathrm{~S}_{8} \mathrm{M}\right]^{+}, \quad\left[\mathrm{C}_{20} \mathrm{H}_{40} \mathrm{Cl}_{3} \mathrm{~N}_{4} \mathrm{~S}_{8} \mathrm{M}_{2}\right]^{+}$, and $\left[\mathrm{C}_{20} \mathrm{H}_{40} \mathrm{Cl}_{4} \mathrm{~N}_{4} \mathrm{~S}_{8} \mathrm{M}_{2}+\mathrm{H}^{+}\right]^{+}$. However, 1:4 complexes of TETD show $\left[\mathrm{C}_{40} \mathrm{H}_{80} \mathrm{~N}_{8} \mathrm{~S}_{16} \mathrm{M}\right]^{2+}$. The complex 5 was also studied by ESI-MS (Fig. 1 in supplementary file). ESI- MS spectra of complex 5 exhibit the signals at 236.7, 238.7, 309.02, 355.0, 384.96, 460.91, 462.91, 532.99, 591.07,
$\left[\mathrm{C}_{20} \mathrm{H}_{40} \mathrm{Cl}_{3} \mathrm{~N}_{4} \mathrm{~S}_{8} \mathrm{Hg}_{2}\right]^{+}, \quad\left[\mathrm{C}_{20} \mathrm{H}_{40} \mathrm{Cl}_{4} \mathrm{~N}_{4} \mathrm{~S}_{8} \mathrm{Hg}_{2}\right]^{+}, \quad\left[\mathrm{C}_{20} \mathrm{H}_{40}\right.$ $\left.\mathrm{Cl}_{4} \mathrm{~N}_{3} . \mathrm{N}^{15} \mathrm{~S}_{8} \mathrm{Hg}_{2}\right]^{+}$, respectively.

Molar conductance values for all 2:2 complexes of dilute concentration $(\sim 5.0 \mathrm{mmol})$ were in the range 10.0-20.6 $\Omega^{-1} \mathrm{~cm}^{2} \mathrm{~mol}^{-1}$ suggesting non-electrolytic nature of the complexes. However, in 1:4 complexes, molar conductance values were quite higher and in the range 160-180 $\Omega^{-1} \mathrm{~cm}^{2} \mathrm{~mol}^{-1}$, indicating 1:2 electrolytic natures of the complexes.

According to FT-IR spectra of ligand (Fig. 2 in supplementary file), sharp two absorption bands exhibited at 991 and $1,046 \mathrm{~cm}^{-1}$, assigned for $v(\mathrm{C}=\mathrm{S})$ were found shifted to lower frequency region and observed at about 912 and $983 \mathrm{~cm}^{-1}$ in the complexes, indicating coordination of metal to sulphur of the thiocarbonyl moiety (Nabeel 
et al. 2005). The complexes $\mathbf{1}, \mathbf{3}$ and $\mathbf{5}$ display bands characteristic of coordinated tetraethylthiuram disulphide (disulphiram), strong absorption in between 1,433-1,545 and $912-987 \mathrm{~cm}^{-1}$ due to $v(\mathrm{C}-\mathrm{N})$ and $v(\mathrm{C}=\mathrm{S})$ modes, which indicate the presence of bidentate bridging nature of disulfiram ligand in these complexes. A shift to higher frequency in the $v(\mathrm{C}-\mathrm{N})$ bands of the binuclear complexes as compared to the ligand indicates the increase in double bond character of the $\mathrm{C}-\mathrm{N}$ bond.

In ${ }^{1} \mathrm{H}-\mathrm{NMR}$ spectra of the complexes (Fig. 3 in supplementary file), triplet ascribed for the $-\mathrm{CH}_{3}$ proton and quartet assigned for $-\mathrm{CH}_{2}$ proton in ligand were almost unshifted and observed at same $\delta$ value.

In ${ }^{13} \mathrm{C}$-NMR spectra of complexes (Fig. 4 in supplementary file), a signal observed at about $\delta 192.56 \mathrm{ppm}$ for thiocarbonyl carbon in ligand was shifted to lower field and appeared at about $\sim \delta 200 \mathrm{ppm}$ in the complexes indicating coordination of ligand to metal by donating lone pair from sulphur of the thiocarbonyl moiety. In all the complexes, signal assigned for methyl carbon $\left(-\mathrm{CH}_{3}\right)$ and methylene carbon $\left(\mathrm{CH}_{2}\right)$ was observed at the same position as it was observed in their corresponding ligand.

Therefore, on the basis of molar conductance, elemental analyses, FT-IR, ${ }^{1} \mathrm{H}-\mathrm{NMR},{ }^{13} \mathrm{C}-\mathrm{NMR}$ FAB-mass and ESImass, the most probable structure for the complexes was suggested as in Figs. 1 and 2.

\section{Characterization of nanoparticles}

\section{$U V$-visible spectra of CdS nanoparticles}

UV-Vis absorption spectroscopy is an efficient technique to monitor the optical properties of quantum-sized particles. It can be used as a convenient method of characterizing the size and size distribution of semiconductor nanoparticles. Semiconductor nanocrystallite are known to have an absorption edge, which is shifted with respect to the bulk material towards shorter wavelengths. This blue shift is thus taken as an indication of the presence of such nanocrystals. The UV-Vis spectra of CdS nanoparticles were recorded in transmission mode and samples were prepared by making dispersion in toluene. For the bulk crystallites, we usually observe the interband absorption spectrum with a band edge around $515 \mathrm{~nm}$. As the crystalline size decreases there is a blue shift of the absorption edge. In the present case, the band edge is observed at $406 \mathrm{~nm}$ with a further shoulder at about $380 \mathrm{~nm}$ realizing that our CdS nanoparticles' sample may not be strictly monodispersed. A similar feature for the UV-Vis spectrum of the nanocrystalline CdS has been reported by Motte (1992) and Hoyer et al. (1995).

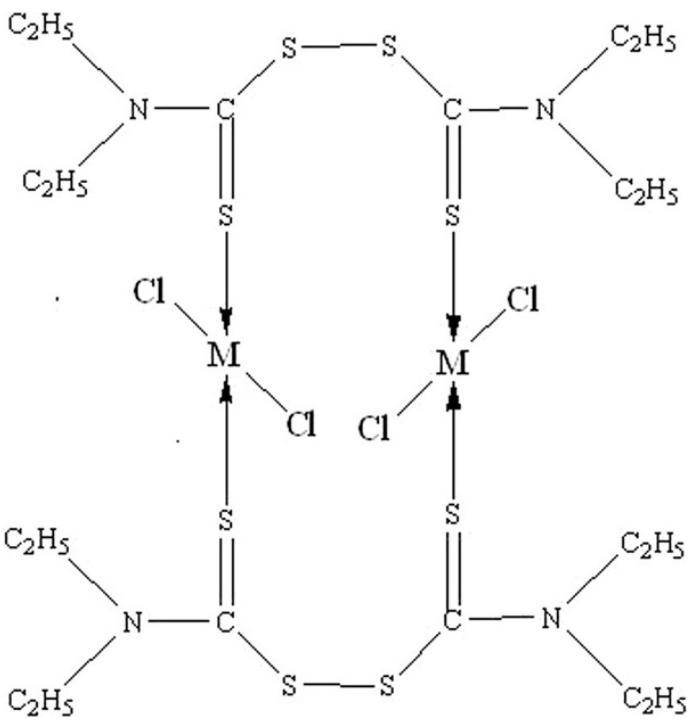

Fig. 1 Structure of complexes 1, 3 and 5, where $\mathrm{M}$ : $\mathrm{Zn}, \mathrm{Cd}$ and $\mathrm{Hg}$

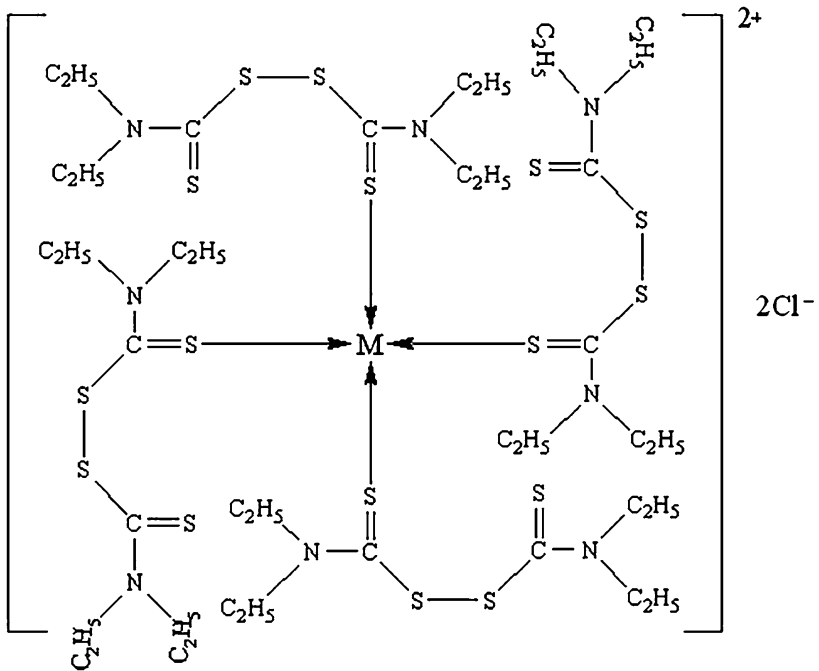

Fig. 2 Structure of complexes 2, 4 and 6, where $\mathrm{M}: \mathrm{Zn}, \mathrm{Cd}$ and $\mathrm{Hg}$

\section{$X$-ray diffraction (XRD) measurements}

XRD pattern provides information about crystalline phase of the nanoparticles as well as the crystallite size (Nair et al. 2002). Mean crystallite size was also calculated using Scherrer's formula (Manickathai et al. 2008). In XRD pattern of ZnS (Fig. 3), prominent peaks were obtained at $26.5417^{\circ}, 32.978^{\circ}$ and $28.2578^{\circ}$. The calculated corresponding d-spacings are 3.36, 2.71 and 3.16 indicating the presence of (100), (101) and (002) reflection plane and hexagonal phase of nanoparticles. The estimated average crystalline grain size of $\mathrm{ZnS}$ sample is $3.6 \mathrm{~nm}$.

The XRD of CdS nanoparticles (Fig. 4) exhibits prominent peaks at $28.2297^{\circ}, 24.8538^{\circ}$ and $26.5417^{\circ}$ corresponding to calculated d-spacings $3.16,3.58$ and 3.35 , 


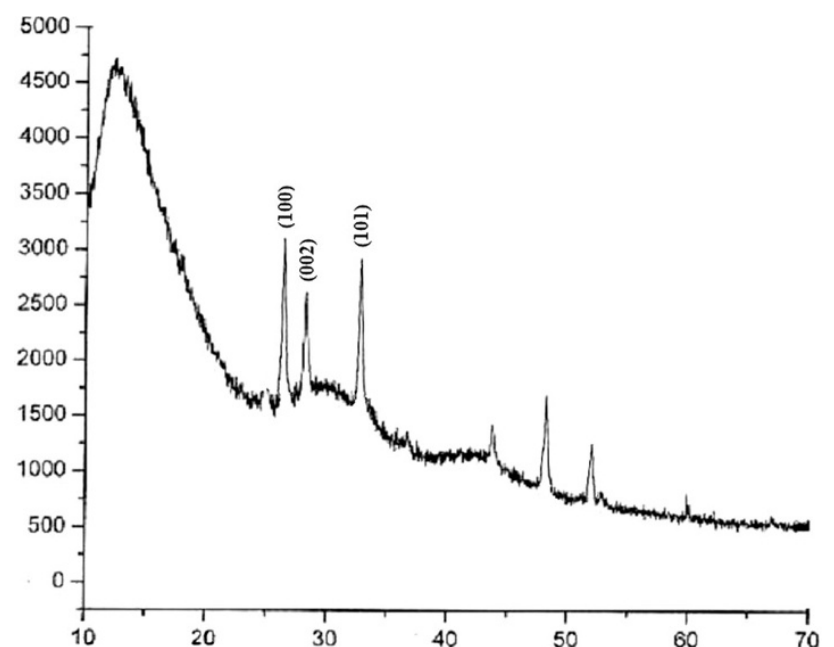

Fig. 3 XRD pattern of $\mathrm{ZnS}$ nanoparticles

respectively, indicating the presence of (101), (100) and (002) reflection planes in accordance with hexagonal phase. The XRD of CdS also exhibits peaks at $43.7586^{\circ}$, $48.9222^{\circ}$ and $51.8888^{\circ}$ corresponding to d-spacings 2.06 , 1.89 and 1.76 indicating the presence of (110), (103) and (112) reflection planes also supports the existence of hexagonal phase (Yadav et al. 2009; Kandasamy et al. 2009). The estimated average grain size of sample is $16.12 \mathrm{~nm}$ obtained from the FWHM of most intense peak.

In XRD pattern of $\mathrm{HgS}$ nanoparticles (Fig. 5), prominent peaks were obtained at $26.4433^{\circ}, 28.1663^{\circ}$ and $43.8219^{\circ}$ corresponding to d-spacings $3.37,3.17$ and 2.06 indicating the presence of (101), (012) and (104) reflection planes. The estimated mean grain size of the sample is $5.78 \mathrm{~nm}$.

\section{Transmission electron microscopy}

TEM allows the direct imaging of nanoparticles and provides more information on the quality of individual particles, e.g. their size, distribution and shape (Hambrock et al. 2001). The TEM image of $\mathrm{ZnS}$ nanoparticles (Fig. 6a) shows quite well-defined particles with narrow size distribution between 5.6 and $16.9 \mathrm{~nm}$ with a mean particle size of $8.9 \mathrm{~nm}$ (see Table 1). The selective area electron diffraction pattern (SAED) for $\mathrm{ZnS}$ exhibits broad diffused rings that are typical of nano-sized particles and a representative pattern is shown in Fig. 6b. The d-spacing values calculated from the SAED pattern are 3.552, 3.205 and 2.853, which are in a good agreement with the XRD results. The size distribution graph is shown in (Fig. 6c).

The TEM image of CdS nanoparticles (Fig. 7a) shows quite well-defined hexagonal particles with a narrow size distribution from 13.3 to $37.5 \mathrm{~nm}$ and mean particle size $26.46 \mathrm{~nm}$ (see Table 1). The SAED for CdS exhibits broad

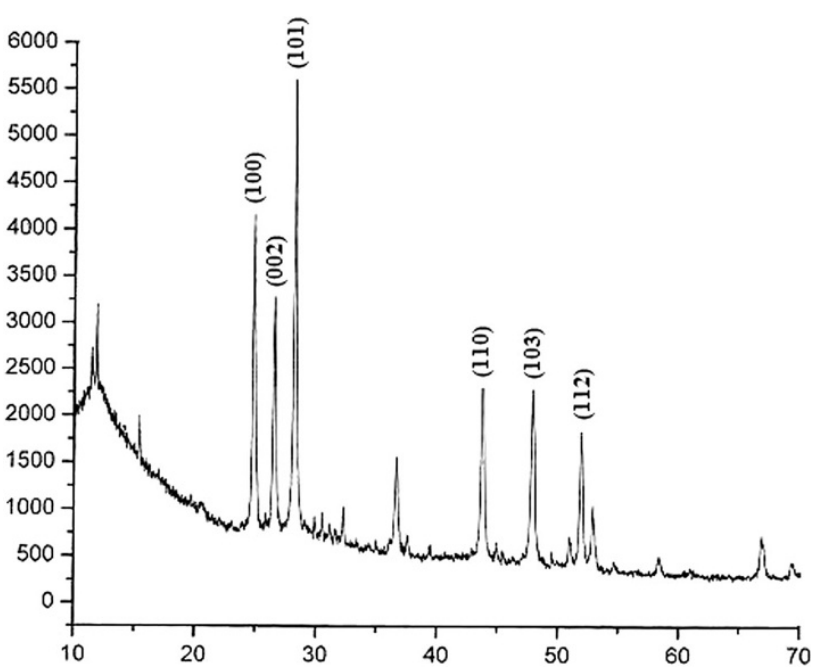

Fig. 4 XRD pattern of CdS nanoparticles

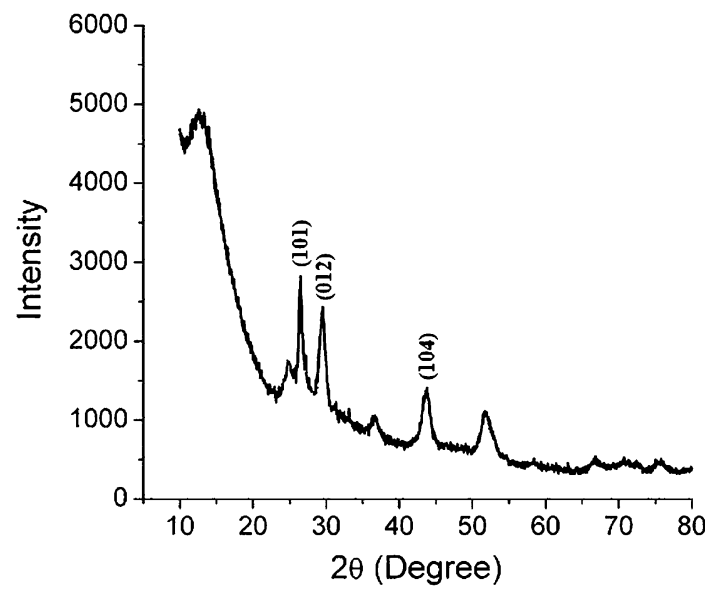

Fig. 5 XRD pattern of $\mathrm{HgS}$ nanoparticles

diffused rings that are typical of nano-sized particles (Fig. 7b). The d-spacing values calculated from the SAED pattern are 3.46, 2.09 and 1.80, which are in a good agreement with the XRD results. The size distribution graph is shown in Fig. 7c.

The TEM image of $\mathrm{HgS}$ nanoparticles (Fig. 8a) shows quite well-defined hexagonal particles with a narrow size distribution from 5.8 to $15 \mathrm{~nm}$ and mean particle size $9.2 \mathrm{~nm}$ (see Table 1). The SAED for $\mathrm{HgS}$ exhibits broad diffused rings that are typical of nano-sized particles and a representative pattern is shown in Fig. 8b. The d-spacing values calculated from the SAED pattern are 3.508, 2.114 and 1.780 , which are in a good agreement with the XRD results. The size distribution graph is shown in Fig. 8c.

\section{Antibacterial activity}

All the metal complexes from 1 to 6 , ligand TETD and the nanoparticles of $\mathrm{CdS}, \mathrm{ZnS}$ and $\mathrm{HgS}$ were screened for 
Fig. 6 a TEM image of $\mathrm{ZnS}$ nanoparticles. b SAED pattern of $\mathrm{ZnS}$ nanoparticles.

c Distribution graph of different size of $\mathrm{ZnS}$ nanoparticles
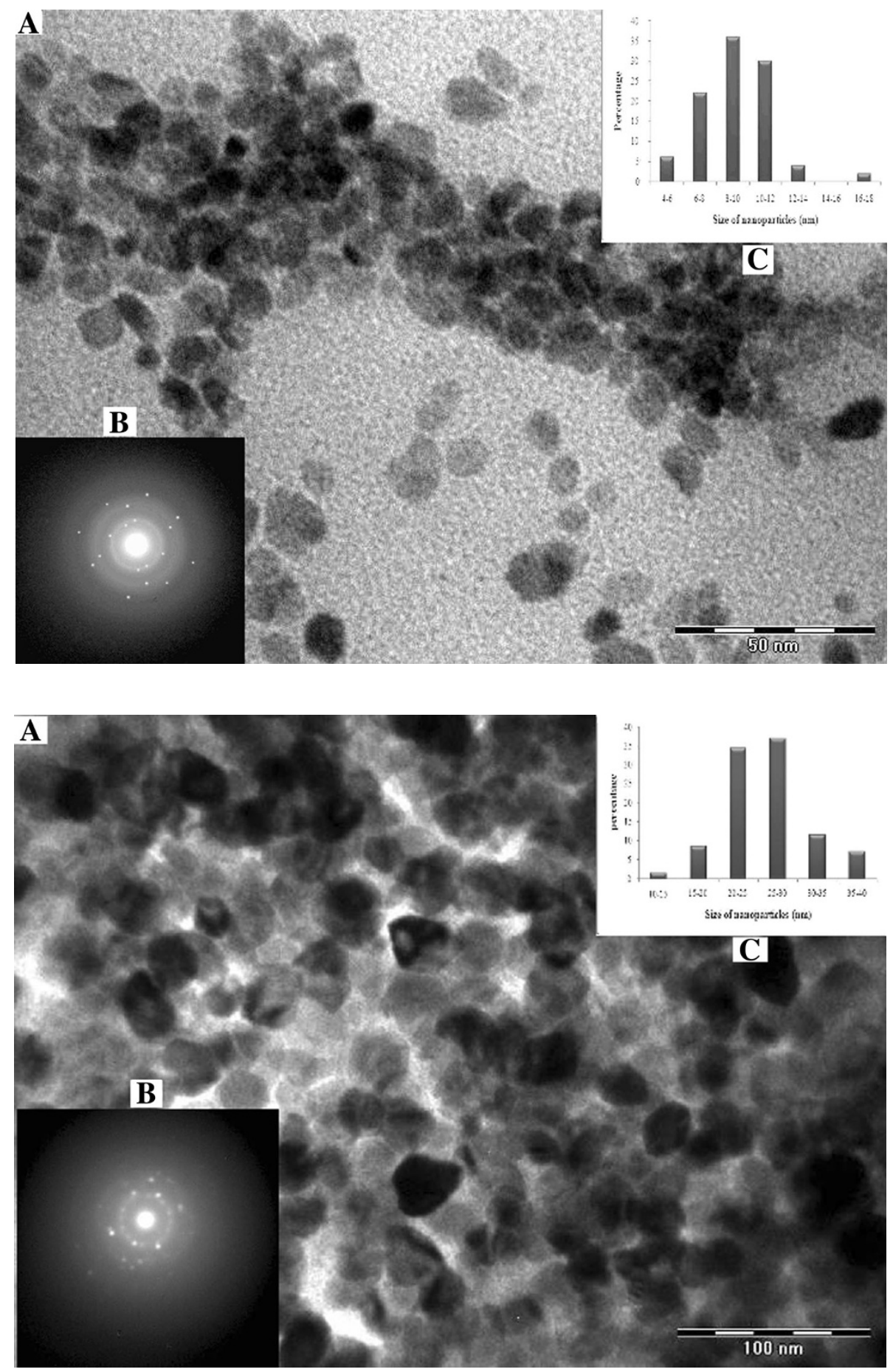

Fig. 7 a TEM image of CdS nanoparticles. b SAED pattern of CdS nanoparticles. c Distribution graph of different size of CdS nanoparticles antibacterial activity against Gram-negative bacteria E. coli, MTCC 1304 at different concentrations. MHA were prepared and $50 \mu \mathrm{L}$ suspensions of $E$. coli containing $\sim 10^{5}$ CFU (colony-forming unit) were applied to the plate by the well-diffusion method (Pelczar et al. 2001). The wells were made on the plates and they were filled with $50 \mu \mathrm{L}$ of sample solution of $0.02 \%$ concentration except in nanoparticles, where $0.02 \%$ dried samples were introduced as such in the well. The $0.02 \%$ solution of chloramphenicol was used for comparison. These plates were incubated at $37 \pm 1{ }^{\circ} \mathrm{C}$ for $24-48 \mathrm{~h}$ in refrigerated incubator shakers. The results in the form of zone inhibition were measured in millimetres. It was observed that all metal complexes show more activity in comparison to ligand, probably due to enhanced lipophilicity of the complexes, which leads to the breakdown of permeability barrier of the cell and thus retards the normal cell process in bacteria. It is postulated that improved activity arises 
Fig. 8 a TEM image of $\mathrm{HgS}$ nanoparticles. b SAED pattern of $\mathrm{HgS}$ nanoparticles. c Distribution graph of different size of $\mathrm{HgS}$ nanoparticles

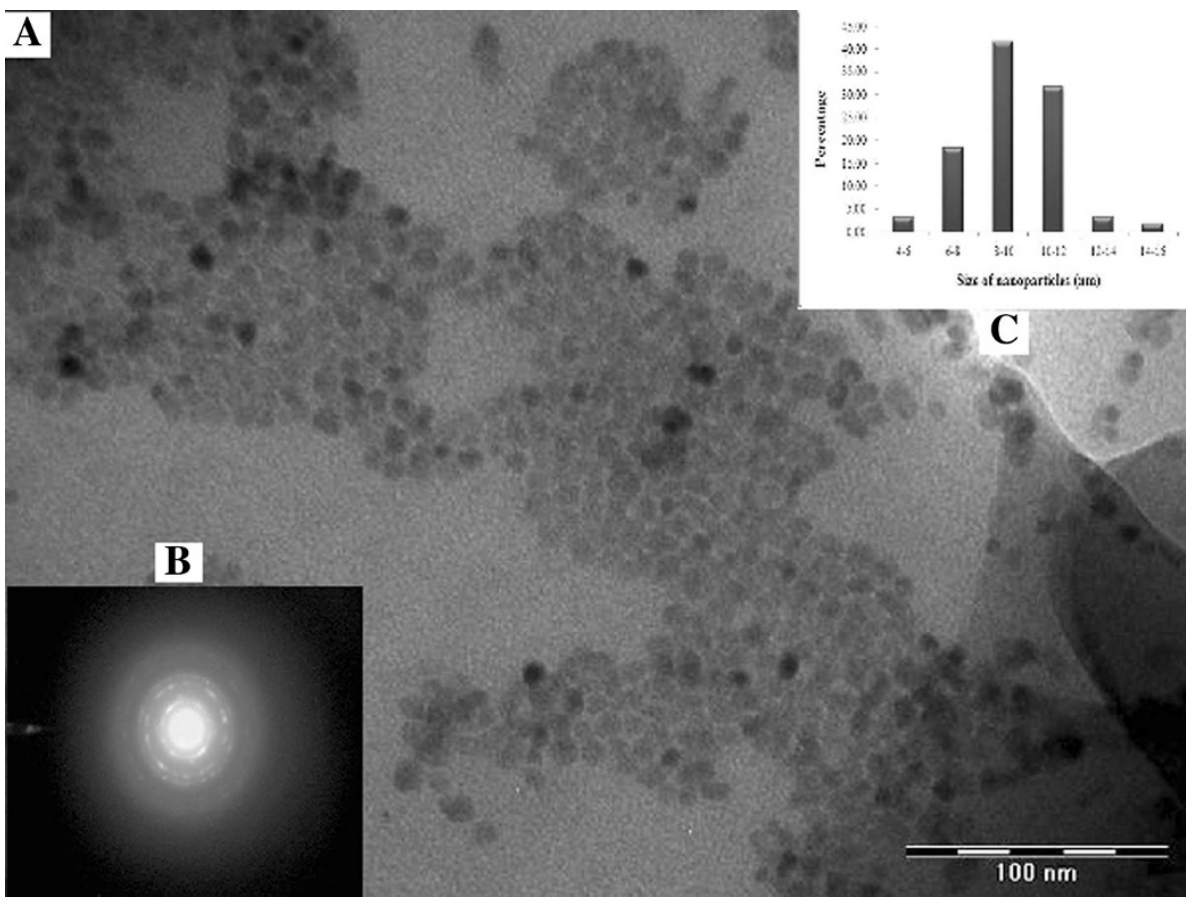

from the delocalization of positive charge between the organic moiety and the metal, which favours the drug entering into normal cellular processes of the bacteria.

The increased activity of metal complexes can be explained on the basis of 'Overtone's concept' and 'Chelation theory'. According to 'Overtone's concept' of cell permeability, the lipid membrane that surrounds the cell favours the passage of only lipid-soluble materials due to which liposolubility is an important factor that controls the antimicrobial activity. On Chelation, the polarity of metal ion will reduce to a greater extent due to overlap of the ligand and metal orbital and partial sharing of positive charge of the metal ion with donor groups.Further, it increases the delocalization of $\pi$-electrons over the whole chelate ring, which enhance the lipophilicity of the complexes into lipid membrane and blocks the metal binding sites on enzyme of pathogenic microorganism. However, it was interestingly observed that nanoparticles exhibited more activity than Schiff base but less than corresponding complexes (see Figs. 5 and 6 of supplementary file). The detail result of antibacterial screening for $0.02 \%$ concentration is given in Table 2 .

\section{Minimum inhibitory concentration (MIC)}

To monitor the activity and confirm their sensitivity, complexes from 1 to 6 and nanoparticles were tested for MIC by successive dilution method (Mazzola et al. 2009).

In six numbered screw tubes $\mathbf{A}-\mathbf{F}(10 \times 100 \mathrm{~mm})$, $1 \mathrm{~mL}$ of Mueller-Hinton broth medium was distributed in each tube, except for the tube A. All the tubes were placed at autoclave for sterilization. In tubes $\mathbf{A}$ and $\mathbf{B}, 1 \mathrm{~mL}$ of test solution (1-6) was added; tube $\mathbf{B}$ was stirred and $1 \mathrm{~mL}$ mixture of the same was taken out and transferred to tube C. The $0.1 \mathrm{~mL}$ suspension of inoculums E. coli, MTCC 1304 having $\sim 500,000$ cells per $\mu \mathrm{L}$ was added to all tubes. Incubation at $37 \pm 1{ }^{\circ} \mathrm{C}$ temperature was maintained for 24-48 h. MIC is concentration of the higher dilution tube, in which bacterial growth was absent and the results are presented in Table 2. It was observed that complex 3 was the most active to inhibit bacteria with inhibition zone of $42 \mathrm{~mm}$ and minimum inhibitory concentration of $0.35 \mu \mathrm{g} /$ $\mathrm{mL}$ for E. coli. Mercury complexes 5 and $\mathbf{6}$ and their nanoparticles have lower MIC value in between 0.20 and $0.25 \mu \mathrm{g} / \mathrm{mL}$. However, it was interestingly observed that MIC of metal sulphide nanoparticles is lower than in corresponding metal complexes, which may be attributed to the increased ratio of surface area to volume in the nanoparticles.

\section{Conclusions}

Six complexes of $\mathrm{Zn}(\mathrm{II}), \mathrm{Cd}(\mathrm{II})$ and $\mathrm{Hg}(\mathrm{II})$ with ligand TETD have been synthesized and characterized by spectroscopic methods. These complexes along with surfactant in the ratio ranging between $1: 1$ and 1:5 for precursor:surfactant were used as single-source precursor for the thermal/pyrolytic synthesis of $\mathrm{ZnS}, \mathrm{CdS}$ and $\mathrm{HgS}$ nanoparticles. Interestingly, it was observed that, at low concentration of precursor and high concentration of surfactant the nanoparticles are hexagonal in shape and smaller in 
size. In synthesis of nanoparticles 1:2, precursor:surfactant ratio is most favourable. At high temperature, agglomeration takes place and large particle size is favoured. In this work, Schiff base complexes were probably for the first time used as single-source precursor for the synthesis of metal sulphide nanoparticles. All the complexes were screened for antibacterial activity against $E$. coli and found more potent than Schiff base ligands and exhibit approximately same toxicity as standard drug. However, nanoparticles exhibited more antibacterial activity than Schiff bases but less than corresponding complexes. It can be concluded that nanoparticles exhibited potency due to their greater surface area, but the synergistic effect of toxophoric functions is more prominent in the complexes, due to which probably complexes are more potent than nanoparticles. Interestingly, nanoparticles exhibit lower MIC than complexes probably due to their higher surface activity.

Acknowledgments We are thankful to our Principal, Govt. Model Science College, Jabalpur and Head, Department of Chemistry, Govt. Model Science College, Jabalpur, (MP) for providing laboratory facilities. One of us (SNS) is thankful to UGC for the sanction of Major Research Project F No. 39-839/2010(SR). We are indebted to Dr. Mukul Gupta Scientist-E, DAE-UGC Consortium Research Center, Indore for his kind help rendered in XRD measurements. We are also thankful to Dr. N. P. Lalla, Scientist-F, DAE-UGC Consortium Research Center, Indore for his kind help in TEM measurements. Thanks are also due to SAIF, CDRI, Lucknow for CHNS analyses, ${ }^{1} \mathrm{H}-\mathrm{NMR}$ and ESI-MS/FAB-MS Spectra.

Open Access This article is distributed under the terms of the Creative Commons Attribution License which permits any use, distribution, and reproduction in any medium, provided the original author(s) and the source are credited.

\section{References}

Bouma MJ, Snowdon D, Fairlamb AH, Ackers JP (1998) Activity of disulfiram (bis(diethylthiocarbamoyl)disulphide) and ditiocarb (diethyldithiocarbamate) against metronidazole-sensitive and -resistant Trichomonas vaginalis and Tritrichomonas foetus. J Antimicrob Chemother 42(6):817-820

Brus LE (1991) Quantum crystallites and nonlinear optics. Appl Phys A Mater Sci Process 53:465-474

Cowley AR, Davis J, Dilworth JR, Donnelly PS, Dobson R, Nightingale A, Peach JM, Shore B, Kerr D, Seymour L (2005) Fluorescence studies of the intra-cellular distribution of zinc bis(thiosemicarbazone) complexes in human cancer cells. Chem Commun (7):845-847

Dahan M, Levi S, Luccardini C, Rostaing P, Riveau B, Triller A (2003) Diffusion dynamics of glycine receptors revealed by single-quantum dot tracking. Science 302:442-445

Hambrock J, Birkner A, Fischer RA (2001) Synthesis of CdSe nanoparticles using various organomettalic cadmium precursors. J Mater Chem 11:3197-3201

Hoffmann MR, Martin ST, Choi WY, Bahnemann DW (1995) Environmental Applications of Semiconductor Photocatalysis. Chem Rev 95:69-96
Hoyer P, Baba N, Masuda H (1995) Small quantum-sized CdS particles assembled to form a regularly nanostructured porous film. Appl Phys Lett 66:2700-2702

Kaczmareka MT, Jastrzaba R, Holderna-Kedziab E, Radecka-Paryzek W (2009) Inorg Chim Acta 362:3127-3133

Kandasamy K, Singh HB, Kulshreshtha SK (2009) Synthesis and characterization of $\mathrm{CdS}$ and $\mathrm{CdSe}$ nanoparticles prepared from novel intramolecularly stabilized single-source precursors. J Chem Sci 121:293-296

Kawamoto T, Nishiwaki M, Tsunekawa Y, Nozaki K, Konno T (2008) Synthesis and characterization of luminescent zinc(II) and cadmium(II) complexes with N,S-chelating Schiff base ligands. Inorg Chem 47:3095-3104

Koike T, Takashige M, Kimura E, Fujioka H, Shiro M (1996) Bis(ZnII-cyclen) Complex as a Novel Receptor of Barbiturates in Aqueous Solution. Chem Eur J 2:617-623

Lidke DS, Nagy P, Heintzmann R, Arndt-Jovin DJ, Post JN, Grecco HE, Jares-Erijman EA, Jovin TM (2004) Quantum dot ligands provide new insights into erbB/HER receptor-mediated signal transduction. Nat Biotechnol 22:198-203

Lobler M, Rohm HW, Schmitz K-P, Johnston AH, Newman TA, Ranjan S, Sood R, Kinnunen PKJ (2009) Drug delivery by nanoparticles-facing the obstacles. In: Proceedings of the 4th European conference of the international federation for medical and biological engineering (IFMBE), vol 22. Springer, Belgium, pp 2335-2338

Manickathai K, Viswanathan SK, Alagar M (2008) Synthesis and characterization of $\mathrm{CdO}$ and $\mathrm{CdS}$ nanoparticles. Indian J Pure Appl Phy 46:561-564

Marchettic F, Pettinari C, Pettinari R, Cingolani A, Leanesi D, Lorenotti A (1999) Group 12 metal complexes of tetradentate $\mathrm{N}_{2} \mathrm{O}_{2}$-Schiff-base ligands incorporating pyrazole: synthesis, characterisation and reactivity toward S-donors, N-donors, copper and tin acceptors. Polyhedron 18:3041-3050

Mazzola PG, Jozala AF, Novaes LCL, Moriel P, Penna TCV (2009) Minimal inhibitory concentration (MIC) determination of disinfectant and/or sterilizing agents. Braz J Pharm Sci 45(2):241-248

Motte L (1992) Synthesis of cadmium sulfide in situ in cadmium bis(2-ethylhexyl) sulfosuccinate reverse micelle: polydispersity and photochemical reaction. Langmuir 8:1049-1053

Nabeel H, Amena FB, Al-Omari (2005) Raf J Sci 16(3):138-145

Nair PS, Radhakrishnan T, Revaprasadu N, Kolawole G, O'Brien P (2002) Cadmium ethylxanthate: A novel single-source precursor for the preparation of CdS nanoparticles. J Mater Chem 12:2722-2725

Nash T, Rice WG (1998) Efficacies of zinc-finger-active drugs against Giardia lamblia. Antimicrob Agents Chemother 42(6): 1488-1492

Onwudiwe DC, Ajibadercury PA (2011) ZnS, CdS and $\mathrm{HgS}$ Nanoparticles via Alkyl-Phenyl Dithiocarbamate Complexes as Single Source Precursors. Int J Mol Sci 12:5538-5551

Pelczar MJ, Chan ECS, Krieg NR (2001) Text book of microbiology, 5th edn. McGraw-Hill Publishing Company Ltd, New Delhi (138)

Sheng X, Guo X, Lu XM, Lu GY, Shao Y, Liu F, Xu Q (2008) DNA binding, cleavage and cytotoxic activity of the preorganized dinuclear $\mathrm{Zn}(\mathrm{II})$ complex of triazacyclononane derivatives. Bioconjugate Chem 19:490-498

Wu MX, Liu H, Liu J, Haley KN, Treadway JA, Larson JP, Ge N, Peale F, Bruchez MP (2003) Immunofluorescent labeling of cancer marker Her2 and other cellular targets with semiconductor quantum dots. Nat Biotechnol 21:41-46

Yadav RS, Mishra R, Pandey AC (2009) Particle size distribution study by small-angle $\mathrm{X}$-ray scattering technique and photoluminescence property of $\mathrm{ZnO}$ nanoparticles. J Exp Nanosci 4(2):139-146 